\title{
CONTRIBUTION TO THE KNOWLEDGE OF THE LICHEN BIOTA OF BOLIVIA. 8
}

\author{
Adam Flakus, Magdalena Oset, Max Rykaczewski, \\ Ulf SchiefElbein \& Martin KuKwa ${ }^{1}$
}

\begin{abstract}
This paper presents new records of 135 lichen taxa in Bolivia. Two species, Myriotrema laeviusculum (Nyl.) Hale and Graphis tenoriensis Chaves \& Lücking, are new for the Southern Hemisphere; two, Cladonia uncialis (L.) Weber ex F. H. Wigg. and Thelocarpon laureri (Flot.) Nyl., are new for South America; and 27 species are new for Bolivia: Acanthotrema brasilianum (Hale) Frisch, Bulbothrix laevigatula (Nyl.) Hale, B. leprieurii Aubel, Canoparmelia salacinifera (Hale) Hale, Catolechia wahlenbergii (Flot. ex Ach.) Körb., Chapsa alborosella (Nyl.) Frisch, Coccocarpia dissecta Swinscow \& Krog, Graphis apertoinspersa Rivas Plata \& Lücking, G. cinerea Fée, G. globosa (Fée) Spreng., G. haleana R. C. Harris, G. marginata Raddi, G. pilarensis Cáceres \& Lücking, G. striatula (Ach.) Spreng., Hemithecium oryzaeforme (Fée) Staiger, Hypotrachyna protochlorina Sipman, Elix \& T. H. Nash, H. sinuosella Elix, T. H. Nash \& Sipman, H. tariensis Elix, Parmeliella isidiopannosa Jørg., Parmotrema conferendum Hale, P. enteroxanthum Hale, P. laciniellum (Ferraro \& Elix) Blanco et al., P. pilosum (Stizenb.) Krog \& Swinscow, P. recipiendum (Nyl.) Hale, P. ruptum (Lynge) Hale ex DePriest \& Hale, Pyxine pungens Zahlbr. and Xanthoparmelia substenophylloides Hale. Notes on distribution are provided for the species. Bulbothrix goebelii (Zenker) Hale is excluded from the list of Bolivian lichens; the record is referred to B. laevigatula.
\end{abstract}

Key words: biodiversity, biogeography, Etayoa trypethelii, lichenized fungi, Neotropics, South America

Adam Flakus, Laboratory of Lichenology, W. Szafer Institute of Botany, Polish Academy of Sciences, Lubicz 46, $31-512$ Kraków, Poland; e-mail: a.flakus@botany.pl

Ulf Schiefelbein, Blücherstr. 71, 18055 Rostock, Germany.e-mail: ulf.schiefelbein@gmx.de

Martin Kukwa, Magdalena Oset \& Max Rykaczewski, Department of Plant Taxonomy and Nature Conservation, University of Gdańsk, Wita Stwosza 59,80-308 Gdańsk,Poland; e-mails:dokmak@ug.edu.pl,magdalenasyrek@wp.pl,max.rykaczewski@gmail.com

\section{INTRODUCTION}

This paper is the eighth part of the series presenting new data on the diversity and distribution of lichenized fungi in Bolivia - a country with one of South America's richest pools of biodiversity, still insufficiently known (e.g., Flakus \& Wilk 2006; Flakus et al. 2011, 2012a, 2014, 2015). The paper updates our current knowledge of Bolivian lichens, reporting new records of 135 lichens from Bolivia, including two species new for the Southern Hemisphere, two new for South America, and 27 new for Bolivia.

\section{MATERIAL AND METHODS}

The examined specimens are housed at B, KRAM, LPB and UGDA; some duplicates are in the private

\footnotetext{
1 Corresponding author
}

herbarium of A. Flakus. Secondary lichen metabolites were identified by thin-layer chromatography (TLC) following Culberson and Kristinsson (1970) and Orange et al. (2001). Notes on world distribution are included for species recorded as new for Bolivia (asterisked), new for South America (2 asterisks) and the Southern Hemisphere (3 asterisks). For the species known from at least five localities in Bolivia, only new records for departments, provinces and/or protected areas are reported, if not otherwise stated.

The following abbreviations are used: $A F-\mathrm{A}$. Flakus, $J Q-$ J. Quisbert, $M K-$ M. Kukwa, $O P-O$. Plata, $P R$ - P. Rodriguez Flakus, AMBORÓ - Parque Nacional y Área Natural de Manejo Integrado Amboró; APOLOBAMBA - Área Natural de Manejo Integrado Nacional Apolobamba; E. B. BENI - Reserva de la Biosfera Estación Biológica del Beni; CARRASCO - Parque Nacional Carrasco; COTAPATA - Parque Nacional y Área Natural de Manejo Integrado Cotapata; KAA-IYA - Parque 
Nacional y Área Natural de Manejo Integrado Kaa-Iya del Gran Chaco; SAJAMA - Parque Nacional Sajama; TARIQUÍA - Reserva Nacional de Flora y Fauna Tariquía; (abbreviations of protected areas according to SERNAP Bolivia; http://www.sernap.gob.bo).

\section{RESULTS}

\section{*Acanthotrema brasilianum (Hale) Frisch}

This species is previously known from Brazil, Colombia, Costa Rica, Peru and Venezuela (Frisch 2006; Cáceres 2007; Sipman et al. 2008, 2012; Rivas Plata \& Lücking 2013).

It was also reported from Africa (Cameroon and Tanzania; Frisch 2006) but according to Rivas Plata et al. (2010) those records belong to A. frischii Lücking.

Specimens examined. BOliVia. Dept. Beni. Prov. Itenez, El Prado colony near Bella Vista, by Río San Martín, $13^{\circ} 17^{\prime} 46^{\prime \prime} \mathrm{S}, 63^{\circ} 35^{\prime} 21^{\prime \prime} \mathrm{W}, 155 \mathrm{~m}$, lowland Amazon forest, corticolous, 15 Aug. 2008, AF 11989, 11964 \& PR (KRAM, LPB); DePt. Santa Cruz. Prov. Ichilo,

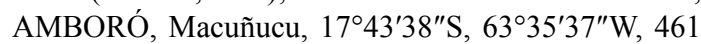
$\mathrm{m}$, lowland Amazon forest, corticolous, 6 June 2011, $A F$ 22297, $22312 \& O P$ (KRAM, LPB).

\section{Alectoria ochroleuca (Hoffm.) A. Massal.}

The species has been reported only rarely from Bolivia (Flakus et al. 2012a, 2015 and literature cited therein). New for Tiraque Province and Parque Nacional Carrasco.

Specimen examined. BOlivia. Dept. CochaBamba. Prov. Tiraque, CARRASCO, Cerro Juno,

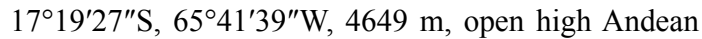
vegetation, terricolous, 29 Nov. 2014, $A F 25871$ (LPB).

\section{Bulbothrix coronata (Fée) Hale}

In Bolivia known previously from a single locality (Flakus et al. 2011).

Specimens examined. BOLIVIA. Dept. Beni. Prov. Ballivan, between Yucumo and Rurrenabaque, $15^{\circ} 05^{\prime} \mathrm{S}$, $67^{\circ} 07^{\prime} \mathrm{W}, 600 \mathrm{~m}$, corticolous, 31 June 1997, K. Bach 363 c et al. (B); Dept. La Paz. Prov. Franz Tamayo, between Apolo and Mapiri, $14^{\circ} 41^{\prime} 50^{\prime \prime} \mathrm{S}, 68^{\circ} 25^{\prime} 07^{\prime \prime} \mathrm{W}$, $1490 \mathrm{~m}$, savannah with scattered trees, corticolous, 22 Nov. 2011, MK 10236a (LPB).

\section{${ }^{*}$ Bulbothrix laevigatula (Nyl.) Hale}

This widespread species is known from Africa (Angola), Asia (Japan, Philippines, Thailand), North America (Mexico, United States), Central America and the Carribean (e.g., Cuba, Dominica, Domican Republic, Jamaica, Saint Vincent, Trinidad and Tobago), and South America (Brazil, Colombia, Ecuador including the Galapagos Islands, French Guiana, Peru, Venezuela) (Hale 1976a; Sipman et al. 2008; Bungartz et al. 2013; Benatti 2014).

According to Benatti (2014) the species contains only lecanoric acid in the thallus medulla. In our material we also detected gyrophoric acid, but in minor amounts. The presence of gyrophoric acid makes our material resemble $B$. papyrina (Fée) Hale but the latter never contains lecanoric acid (Benatti 2014).

Flakus et al. (2011) reported one of the specimens cited below as B. goebeli (Zenker) Hale, but revision of its type indicated that this taxon contains lobaric acid and lacks isidia (Benatti \& Elix 2012), so the Bolivian record belongs to B. laevigatula.

Specimen examined. BOLIVIA. Dept. CochaBAMBA. Prov. Chapare, near Bulo Bulo village, near Río Chimoré, $16^{\circ} 58^{\prime} 19^{\prime \prime} \mathrm{S}, 65^{\circ} 21^{\prime} 11^{\prime \prime} \mathrm{W}, 283 \mathrm{~m}$, lowland Amazon forest, on bark, 9 Dec. 2010, $A F 19500 \& J Q$ (KRAM, LPB); DePT. La PAZ. Prov. Larecaja, near Achiquiri, $18 \mathrm{~km}$ from Mapiri on road to Apolo, 15 ${ }^{\circ} 13^{\prime} 31^{\prime \prime} \mathrm{S}$, $68^{\circ} 13^{\prime} 49^{\prime \prime} \mathrm{W}, 1070 \mathrm{~m}$, Yungas inferior secondary forest, corticolous, 16 May 2011, MK 8646 (LPB, UGDA).

\section{*Bulbothrix leprieurii Aubel}

Found previously in Brazil, Colombia and Guyana (Sipman et al. 2008; Benatti 2014).

Specimens examined. BOliVia. Dept. La Paz. Prov. Franz Tamayo, between Apolo and Mapiri, $15^{\circ} 01^{\prime} 50^{\prime \prime} \mathrm{S}, 68^{\circ} 18^{\prime} 33^{\prime \prime} \mathrm{W}, 1120 \mathrm{~m}$, Preandean Amazon forest, on twig, 23 Nov. 2011, MK 10332 (LPB, UGDA).

Canoparmelia amazonica (Nyl.) Elix \& Hale

Fourth Bolivian record of the species (Hale 1976b; Flakus et al. 2011, 2013).

Specimens examined. BOliVia. Dept. La Paz. Prov. Franz Tamayo, between Apolo and Mapiri, 13 km 
from Apolo, 1450'51"S, 68²1'38"W, 1490 m, savannah with scattered trees, shrubs, along streams, corticolous, 23 Nov. 2011, MK 10291 (LPB, UGDA).

\section{Canoparmelia caroliniana (Nyl.) Elix \& Hale}

In Bolivia reported previously from three localities (Flakus et al. 2012a, 2013).

Specimens examined. BOLIVIA. Dept. Tarija. Prov. Burnet O'Connor, Lomas de la Soledad, road between Entre Ríos and Chiquiacá, $21^{\circ} 39^{\prime} 38^{\prime \prime} \mathrm{S}, 6^{\circ} 07^{\prime} 31^{\prime \prime} \mathrm{W}$, $1670 \mathrm{~m}$, Tucumano-Boliviano altimontano forest, corticolous, 10 Aug. 2012, MK 11298 (LPB, UGDA).

\section{* Canoparmelia salacinifera (Hale) Hale}

Previously reported in the Neotropics from Brazil, Colombia, Cuba, Honduras, Jamaica, Mexico and Venezuela, and also from the northeastern USA and Thailand (Hale 1976b; Sipman et al. 2008; Pérez Pérez \& Nash 2010).

Specimens examined. BOLIVIA. Dept. Santa Cruz. Prov. Cordillera, KAA-IYA, near Peto Blanco, $18^{\circ} 56^{\prime} 26^{\prime \prime} \mathrm{S}, 60^{\circ} 22^{\prime} 39^{\prime \prime} \mathrm{W}, 340 \mathrm{~m}$, Chiquitano forest, corticolous, 5 Dec. 2011, MK 10620 (LPB, UGDA).

\section{${ }^{*}$ Catolechia wahlenbergii (Flot. ex Ach.) Körb.}

A widespread species reported from Asia, Europe and North, Central and South America (Gilbert \& Hawksworth 2009). In South America it is known from Argentina (Calvelo \& Liberatore 2002).

Specimens examined. BOliVia. Dept. CochaBamba. Prov. Tiraque, CARRASCO, Camino de los Nubes-Cotany Alto road, $17^{\circ} 17^{\prime} 28^{\prime \prime} \mathrm{S}, 65^{\circ} 44^{\prime} 05^{\prime \prime} \mathrm{W}$, $4146 \mathrm{~m}$, open high Andean vegetation, terricolous, 2 Dec. 2014, AF 26065 (KRAM, LPB); DePt. La PAZ. Prov. Franz Tamayo, APOLOBAMBA, Paso Sánchez, near Pelechuco-Keara road, $14^{\circ} 44^{\prime} 41^{\prime \prime} \mathrm{S}, 6^{\circ} 06^{\prime} 36^{\prime \prime} \mathrm{W}$, $4780 \mathrm{~m}$, open high Andean vegetation, terricolous, 17 Nov. 2014, $A F 25165$ (LPB).

\section{Cetraria aculeata (Schreb.) Fr.}

In Bolivia reported previously only by Fernández-Mendoza and Printzen (2013).

Specimen examined. BOLIVIA. Dept. Cochabamba. Prov. Tiraque, CARRASCO, Cerro Juno, near Antenas, $17^{\circ} 19^{\prime} 27^{\prime \prime} \mathrm{S}, 65^{\circ} 41^{\prime} 39^{\prime \prime} \mathrm{W}, 4649 \mathrm{~m}$, open high Andean vegetation, terricolous, 29 Nov. 2014, $A F 25872$ (LPB).

\section{Chaenotheca confusa Tibell}

Second Bolivian locality of the species (Flakus et al. 2014).

Specimens examined. BOliVia. Dept. La Paz. Prov. Franz Tamayo, APOLOBAMBA, below Pelechuco, $14^{\circ} 49^{\prime} 08^{\prime \prime} \mathrm{S}, 69^{\circ} 03^{\prime} 50^{\prime \prime} \mathrm{W}, 3560 \mathrm{~m}$, Ceja de Monte Inferior (Altimontano), open area with shrubs and Polylepis trees, on Polylepis sp., 20 Nov. 2014, MK 14672 (LPB, UGDA).

\section{${ }^{*}$ Chapsa alborosella (Nyl.) Frisch}

Previously reported in the Neotropics from Brazil, Colombia, Costa Rica, Peru and Venezuela, and elsewhere from India, Japan, Réunion, Sri Lanka, Tanzania and the USA (Frisch 2006; Cáceres 2007; Sipman et al. 2008, 2012; Rivas Plata \& Lücking 2013).

Specimen examined. BOLIVIA. Dept. La Paz. Prov. Larecaja, between Tipuani and Guanay, $15^{\circ} 31^{\prime} 24^{\prime \prime} \mathrm{S}$, $67^{\circ} 58^{\prime} 06^{\prime \prime} \mathrm{W}, 1200 \mathrm{~m}$, Preandean Amazon forest, corticolous, 24 Nov. 2011, AF 23194 (LPB).

\section{Cladonia confusa $\mathrm{R}$. Sant. f. confusa}

Considered common in Bolivia, but underrecorded (Ahti 2000; Flakus et al. 2008, 2012a, 2014). New for Tarija department.

Specimens examined. BOliVia. Dept. Tarija. Prov. Burnet O'Connor, Lomas de la Soledad, road between Entre Ríos and Chiquiacá, $21^{\circ} 39^{\prime} 38^{\prime \prime} \mathrm{S}, 64^{\circ} 07^{\prime} 31^{\prime \prime} \mathrm{W}$, $1670 \mathrm{~m}$, Tucumano-Boliviano altimontano forest, on ground, 10 Aug. 2012, MK 11253 (LPB, UGDA).

\section{Cladonia dactylota Tuck.}

In Bolivia known previously from several localities (Flakus 2008; Flakus et al. 2008, 2012a). New for Franz Tamayo Province and Área Natural de Manejo Integrado Nacional Apolobamba.

Specimen examined. BOliVia. Dept. La Paz. Prov. Franz Tamayo, APOLOBAMBA, near Rio Pelechuco, below Pelechuco close to new road to Apolo, $14^{\circ} 47^{\prime} 28^{\prime \prime} \mathrm{S}, 69^{\circ} 01^{\prime} 32^{\prime \prime} \mathrm{W}, 2860 \mathrm{~m}$, open area with scattered trees, saxicolous, 16 Nov. 2014, $A F 25137$ (LPB).

\section{Cladonia melanopoda Ahti}

Reported several times from Bolivia (e.g., Ahti 2000; Flakus et al. 2008, 2012a). Here recorded as 
new for Franz Tamayo Province and Área Natural de Manejo Integrado Nacional Apolobamba.

Specimens examined. BOliVia. Dept. La Paz. Prov. Bautista Saavedra, APOLOBAMBA, near la Curva, close to Charazani, $15^{\circ} 06^{\prime} 33^{\prime \prime} \mathrm{S}, 69^{\circ} 01^{\prime} 51^{\prime \prime} \mathrm{W}$, 3634 m, open area with shrubs, on rock, 14 Nov. 2014, MK 14648 (LPB, UGDA).

\section{${ }^{* *}$ Cladonia uncialis (L.) Weber ex F. H. Wigg.}

A common circumpolar species in the Northern Hemisphere, previously known in the Neotropics only from the Dominican Republic (Ahti 2000).

Based on morphology, the Bolivian sample represents $C$. uncialis ssp. uncialis (Stenroos et al. 2015), but molecular study is needed for certain identification.

Specimens examined. BOliVia. Dept. CochaBAmba. Prov. Tiraque, CARRASCO, Cerro Juno, near Antenas, $17^{\circ} 19^{\prime} 27^{\prime \prime} \mathrm{S}, 65^{\circ} 41^{\prime} 39^{\prime \prime} \mathrm{W}, 4649 \mathrm{~m}$, open high Andean vegetation, terricolous, 29 Nov. 2014, AF 25869 (KRAM, LPB).

\section{Coccocarpia adnata Arv.}

In Bolivia reported previously from two localities (Kukwa et al. 2014).

Specimens examined. BOliVia. Dept. La Paz. Prov. Muñecas, APOLOBAMBA, above Camata, close to Charazani-Paujeyuyo road, $15^{\circ} 14^{\prime} 35^{\prime \prime} \mathrm{S}, 68^{\circ} 45^{\prime} 09^{\prime \prime} \mathrm{W}$, 1900 m, open area with shrubs, on shrub, 13 Nov. 2014, MK 14583 (LPB, UGDA).

\section{*Coccocarpia dissecta Swinscow \& Krog}

Known previously from Costa Rica, Paraguay and the Paleotropics (Lücking et al. 2007).

Specimens examined. BOliVia. Dept. La Paz. Prov. Franz Tamayo, APOLOBAMBA, near Paujeyuyo, $15^{\circ} 02^{\prime} 22^{\prime \prime} \mathrm{S}, 68^{\circ} 28^{\prime} 36^{\prime \prime} \mathrm{W}, 1010 \mathrm{~m}$, disturbed Sub-Andean Amazon forest, corticolous, 12 Nov. 2014, $M K$ 14540 (LPB, UGDA); Prov. Nor Yungas, COTAPATA, near Urpuma colony, $16^{\circ} 13^{\prime} 20^{\prime \prime} \mathrm{S}, 6^{\circ} 52^{\prime} 34^{\prime \prime} \mathrm{W}, 1989 \mathrm{~m}$, Yungas montane forest, corticolous, 30 June 2010, $A F$ 17325, PR (LPB).

\section{Coccocarpia domingensis Vain.}

In Bolivia reported previously from three localities (Kukwa et al. 2014).
Specimens examined. BOliVia. Dept. La Paz. Prov. Muñecas, APOLOBAMBA, above Camata, close to Charazani-Paujeyuyo road, $15^{\circ} 14^{\prime} 35^{\prime \prime} \mathrm{S}$, $68^{\circ} 45^{\prime} 09^{\prime \prime} \mathrm{W}, 1900 \mathrm{~m}$, open area with shrubs, on shrub, 13 Nov. 2014, MK 14583 (LPB, as admixture to Coccocarpia adnata).

\section{Coccocarpia epiphylla (Fée) Kremp.}

In Bolivia reported recently from one locality (Kukwa et al. 2014).

Specimens examined. BOLIVIA. Dept. La Paz. Prov. Murillo, Sainani, Valle del Zongo, $16^{\circ} 07^{\prime} 20^{\prime \prime}$ S, $68^{\circ} 05^{\prime} 09^{\prime \prime} \mathrm{W}, 2220 \mathrm{~m}$, open area with shrubs and scattered trees, corticolous, 7 Dec. 2014, AF 26243 (LPB).

\section{Coccocarpia erythroxyli (Spreng.) Swinscow} \& Krog

In Bolivia reported previously from several localities (Feuerer \& Sipman 2005; Flakus 2008; Flakus et al. 2012a; Kukwa et al. 2014). New for Murillo Province.

Specimens examined. BOliVia. Dept. La Paz. Prov. Murillo, Sainani, Valle del Zongo, $16^{\circ} 07^{\prime} 20^{\prime \prime}$ S, $68^{\circ} 05^{\prime} 09^{\prime \prime} \mathrm{W}, 2220 \mathrm{~m}$, open area with shrubs and scattered trees, corticolous, 7 Dec. 2014, AF 26241 (KRAM, LPB).

Coccocarpia pellita (Ach.) Müll. Arg.

In Bolivia reported previously by Ferraro (2002), Lücking (2008), Flakus et al. (2012a) and Kukwa et al. (2014). New for Franz Tamayo Province and Área Natural de Manejo Integrado Nacional Apolobamba.

Specimens examined. BOliVia. Dept. La Paz. Prov. Franz Tamayo, APOLOBAMBA, near Paujeyuyo, $15^{\circ} 02^{\prime} 22^{\prime \prime} \mathrm{S}, 68^{\circ} 28^{\prime} 36^{\prime \prime} \mathrm{W}, 1010 \mathrm{~m}$, disturbed Sub-Andean Amazon forest, corticolous, 12 Nov. 2014, $M K$ 14535 (LPB, UGDA).

Coccocarpia prostrata Lücking, Aptroot \& Sipman

In Bolivia reported only recently (Kukwa et al. 2014).

Specimens examined. BOliVia. Dept. La Paz. Prov. Franz Tamayo, APOLOBAMBA, near Paujeyuyo, $15^{\circ} 02^{\prime} 22^{\prime \prime} \mathrm{S}, 68^{\circ} 28^{\prime} 36^{\prime \prime} \mathrm{W}, 1010 \mathrm{~m}$, disturbed Sub-Andean Amazon forest, corticolous, 12 Nov. 2014, $M K$ 14545 (LPB, UGDA). 


\section{Coenogonium tuckermanii Mont.}

In Bolivia reported only a few times (Nylander 1859; Rivas Plata et al. 2006; Flakus 2008).

Specimens examined. BOliVia. Dept. CochaBAmba. Prov. Chapare, CARRASCO, San Jacinto, close to Villa Tunari-Lago Corani road, $17^{\circ} 10^{\prime} 26^{\prime \prime} \mathrm{S}$, $65^{\circ} 45^{\prime} 13^{\prime \prime} \mathrm{W}, 1877 \mathrm{~m}$, open area with shrubs and scattered trees, corticolous, 4 Dec. 2014, AF 26222 (LPB, herb. Flakus).

\section{Coniocarpon cinnabarinum DC.}

In Bolivia reported recently from six localities (Ertz et al. 2015). The record below is the second one from Carrasco Province.

Specimens examined. BOliVia. Dept. CochaBAmBa. Prov. Carrasco, CARRASCO, near Río Lopez Mendoza, $17^{\circ} 30^{\prime} 25^{\prime \prime} \mathrm{S}, 65^{\circ} 16^{\prime} 51^{\prime \prime} \mathrm{W}, 2248 \mathrm{~m}$, lower montane Yungas cloud forest, corticolous, 27 Nov. 2014, $A F$ 25640, 25638 (KRAM, LPB).

\section{Cora arachnoidea J. E. Hern. \& Lücking}

In Bolivia reported previously from only two localities (Lücking et al. 2013; Flakus et al. 2014).

Specimens examined. BOliVia. Dept. La Paz. Prov. Nor Yungas, below Unduavi, on road between La Paz and Chulumani, $16^{\circ} 18^{\prime} 27^{\prime \prime} \mathrm{S}, 67^{\circ} 53^{\prime} 48^{\prime \prime} \mathrm{W}$, $3210 \mathrm{~m}$, Yungas cloud forest, on bryophytes, 31 May 2011, AF 22219 \& OP (LPB, KRAM); COTAPATA, near Unduavi by Sillu Tincara pre-Columbian route, $16^{\circ} 17^{\prime} 22^{\prime \prime} \mathrm{S}, 67^{\circ} 53^{\prime} 29^{\prime \prime} \mathrm{W}, 3518 \mathrm{~m}$, transition Páramo Yungueño-Yungas cloud forest, on bryophytes, 25 May 2011, $A F 21815 \& O P$ (LPB, KRAM).

\section{Cora ciferrii (Tomas) Lücking, Grall \& Thüs}

C. pavonia auct. non (Sw.) Fr.

Fourth locality of the species in Bolivia (Flakus et al. 2014).

Specimens examined. BOliVia. Dept. La Paz. Prov. Bautista Saavedra, APOLOBAMBA, near la Curva, close to Charazani, $15^{\circ} 06^{\prime} 15^{\prime \prime} \mathrm{S}, 69^{\circ} 00^{\prime} 32^{\prime \prime} \mathrm{W}$, $3928 \mathrm{~m}$, open area with shrubs, on bryophytes, 14 Nov. 2014, $M K 14623$ (LPB, UGDA).

\section{Cora glabrata (Spreng.) Fr.}

Several previous Bolivian records of the species appear to represent other recently distin- guished taxa; the record below is the fourth one from Bolivia (Flakus et al. 2012a, 2014).

Specimens examined. BOliVia. Dept. La Paz. Prov. Bautista Saavedra, APOLOBAMBA, near la Curva, close to Charazani, $15^{\circ} 06^{\prime} 15^{\prime \prime} \mathrm{S}, 69^{\circ} 00^{\prime} 32^{\prime \prime} \mathrm{W}$, $3928 \mathrm{~m}$, open area with shrubs, on bryophytes, 14 Nov. 2014, MK 14624 (LPB, UGDA).

\section{Crocodia aurata (Ach.) Link}

Common in Bolivia (Kukwa et al. 2014). New for Chapare, Carraso and Muñecas provinces and Área Natural de Manejo Integrado Nacional Apolobamba.

Specimens eXamined. BOLIVIA. Dept. CochaBamba. Prov. Chapare, near Incachaca, $17^{\circ} 12^{\prime} 54^{\prime \prime} \mathrm{S}$, $65^{\circ} 49^{\prime} 30^{\prime \prime} \mathrm{W}, 2028 \mathrm{~m}$, Yungas cloud forest near road, corticolous, 8 July 2009, AF 12981 (LPB); Prov. Carrasco, CARRASCO, Meruvia close to Monte Punku, $17^{\circ} 35^{\prime} 06^{\prime \prime} \mathrm{S}, 65^{\circ} 14^{\prime} 54^{\prime \prime} \mathrm{W}, 3283 \mathrm{~m}$, Podocarpus-Polylepis forest, corticolous, 26 Nov. 2014, AF 25555 (LPB); DePt. La Paz. Prov. Muñecas, APOLOBAMBA, above Camata, close to Charazani-Paujeyuyo road, $15^{\circ} 14^{\prime} 18^{\prime \prime} \mathrm{S}$, $68^{\circ} 45^{\prime} 09^{\prime \prime} \mathrm{W}, 1800 \mathrm{~m}$, open area with shrubs, on shrub, 13 Nov. 2014, $M K 14603$ (LPB, UGDA).

\section{Cystocoleus ebeneus (Dillwyn) Thwaites}

In Bolivia reported previously from three localities (Flakus \& Wilk 2006; Flakus 2008).

Specimens examined. BOliVia. Dept. La Paz. Prov. Franz Tamayo, APOLOBAMBA, Paso Sánchez, near Pelechuco-Keara road, $14^{\circ} 44^{\prime} 41^{\prime \prime} \mathrm{S}, 6^{\circ} 06^{\prime} 36^{\prime \prime} \mathrm{W}$, 4780 m, open high Andean vegetation, 17 Nov. 2014, $A F$ 25166 (KRAM, LPB); Prov. Larecaja, near Ancoruma, close to Sorata-Mapiri road, $14^{\circ} 42^{\prime} 48^{\prime \prime} \mathrm{S}, 68^{\circ} 35^{\prime} 36^{\prime \prime} \mathrm{W}$, $4641 \mathrm{~m}$, open high Andean vegetation, bryophylous, 21 Nov. 2014, $A F 25518$ (LPB).

\section{Dibaeis columbiana (Vain.) Kalb \& Gierl}

In Bolivia reported previously from four localities (Gierl \& Kalb 1993; Flakus \& Wilk 2006; Flakus et al. 2012a, 2015).

Specimens examined. BOliVia. Dept. La Paz. Prov. Larecaja, near Consata, $15^{\circ} 20^{\prime} 46^{\prime \prime} \mathrm{S}, 68^{\circ} 29^{\prime} 16^{\prime \prime} \mathrm{W}$, $1240 \mathrm{~m}$, Yungas montano inferior secondary forest, on rock, 15 May 2011, MK 8594 (LPB); Prov. Nor Yungas, on road between La Paz and Chulumani, $16^{\circ} 14^{\prime} 10^{\prime \prime} \mathrm{S}$, $67^{\circ} 44^{\prime} 20^{\prime \prime} \mathrm{W}$, Yungas secondary cloud forest, terricolous, 24 May 2011, MK 9337 (LPB, UGDA). 
Diorygma antillarum (Vain.) Nelsen, Lücking \& Rivas Plata

In Bolivia reported previously from two localities (Schiefelbein et al. 2014).

Specimens examined. BOliVia. Dept. Santa Cruz. Prov. Ichilo, AMBORÓ, Macuñucu, 17043'38"S, $63^{\circ} 35^{\prime} 37^{\prime \prime} \mathrm{W}, 461 \mathrm{~m}$, lowland Amazon forest, corticolous, 6 June 2011, $A F 22303 \& O P$ (KRAM, LPB); Dept TariJa. Prov. Aniceto Arce, Filo de Sidras, $22^{\circ} 14^{\prime} 50^{\prime \prime} \mathrm{S}, 64^{\circ} 33^{\prime} 28^{\prime \prime} \mathrm{W}, 1064 \mathrm{~m}$, Tucumano-Boliviano submontane forest, corticolous, 22 Nov. 2010, AF 18421, 18618, 18674 (KRAM, LPB); near Tarija, $22^{\circ} 14^{\prime} 56^{\prime \prime} \mathrm{S}, 64^{\circ} 32^{\prime} 55^{\prime \prime} \mathrm{W}, 883 \mathrm{~m}$, Tucumano-Boliviano secondary submontane forest, corticolous, 21 Nov. 2010, AF 18343, 18378 (KRAM, LPB).

\section{Flavocetraria cucullata (Bellardi) Kärnefelt} \& Thell.

In Bolivia reported several times but only a few records are recent (Herzog 1922; Hasselroth 1953; Menhofer 1992; Feuerer et al. 1998; Bjerke \& Elvebakk 2004; Flakus et al. 2012a). New for Cochabamba department.

Specimens examined. BOliVia. Dept. Cochabamba. Prov. Tiraque, CARRASCO, Diente del Diablo, close to Antenas Sillar-Cotany Alto road, $17^{\circ} 16^{\prime} 55^{\prime \prime}$, $65^{\circ} 44^{\prime} 14^{\prime \prime} \mathrm{W}, 4335 \mathrm{~m}$, open high Andean vegetation, terricolous, 2 Dec. 2014, AF 26052, MK 14669 (LPB, UGDA).

\section{Glyphis cicatricosa Ach.}

In Bolivia reported previously from several localities (Flakus \& Wilk 2006; Flakus et al. 2013). New for Parque Nacional y Área Natural de Manejo Integrado Kaa-Iya del Gran Chaco.

Specimens examined. BOLIVIA. Dept. Santa Cruz. Prov. Cordillera, KAA-IYA, near Peto Blanco, park guard's station, $18^{\circ} 51^{\prime} 36^{\prime \prime} \mathrm{S}, 60^{\circ} 18^{\prime} 00^{\prime \prime} \mathrm{W}, 290 \mathrm{~m}$, transition Chiquitano-Chaqueño forest, corticolous, 5 Dec. 2011, AF 23725 (KRAM, LPB).

\section{Glyphis scyphulifera (Ach.) Staiger}

Fourth Bolivian record of the species (Flakus et al. 2013; Kukwa et al. 2013).

Specimen examined. BOliVia. Dept. Santa Cruz. Prov. Cordillera, KAA-IYA, Bañados del Izozog, near
Río Parapeti, $18^{\circ} 28^{\prime} 40^{\prime \prime} \mathrm{S}, 62^{\circ} 05^{\prime} 03^{\prime \prime} \mathrm{W}, 320 \mathrm{~m}$, Ribereño forest, corticolous, 2 Dec. 2010, AF $18895 \& J Q$ (LPB).

\section{${ }^{*}$ Graphis acharii Fée}

In Bolivia known previously from only two localities (Kukwa et al. 2013).

Specimens eXamined. BOLIVIA. Dept. Cochabamba. Prov. Chapare, near Incachaca, $17^{\circ} 12^{\prime} 54^{\prime \prime} \mathrm{S}, 65^{\circ} 49^{\prime} 30^{\prime \prime} \mathrm{W}$, $2028 \mathrm{~m}$, Yungas cloud forest near road, corticolous, 7 July 2009, AF 12985 (KRAM, LPB).

${ }^{*}$ Graphis apertoinspersa Rivas Plata \& Lücking

Previously known from the type locality in Peru (Rivas Plata \& Lücking 2013).

Specimens examined. BOLIVIA. Dept. La Paz. Prov. Murillo, Valle del Zongo, $16^{\circ} 07^{\prime} 41^{\prime \prime} \mathrm{S}, 68^{\circ} 05^{\prime} 55^{\prime \prime} \mathrm{W}$, $2446 \mathrm{~m}$, Yungas cloud forest, corticolous, 29 May 2011, $A F 22069$ \& $O P$ (KRAM, LPB).

\section{Graphis argentata Lücking \& Umaña}

In Bolivia reported previously from four localities (Flakus et al. 2013; Kukwa et al. 2013).

Specimens examined. BOLIVIA. Dept. CochaBAmBA. Prov. Chapare, near Incachaca, $17^{\circ} 12^{\prime} 54^{\prime \prime} \mathrm{S}$, $65^{\circ} 49^{\prime} 30^{\prime \prime} \mathrm{W}, 2028 \mathrm{~m}$, Yungas cloud forest near road, corticolous, 7 July 2009, AF 13012, 13013, 13028 (KRAM, LPB).

\section{Graphis cincta (Pers.) Aptroot}

Second Bolivian locality of the species (Schiefelbein et al. 2014).

Specimens examined. BOliVia. Dept. Santa Cruz. Prov. Cordillera, between Tucavaca and Roboré, $18^{\circ} 36^{\prime} 11^{\prime \prime} \mathrm{S}, 59^{\circ} 53^{\prime} 06^{\prime \prime} \mathrm{W}, 320 \mathrm{~m}$, transition ChiquitanoChaqueño forest, corticolous, with Etayoa trypethelii, 4 Dec. 2011, AF 23546 (KRAM, LPB).

\section{${ }^{*}$ Graphis cinerea Fée}

Previously known from Brazil, Colombia, Costa Rica and Venezuela (Staiger 2002; Lücking et al. 2008; Sipman et al. 2008).

Specimens examined. BOliVia. Dept. La Paz. Prov. Nor Yungas, km 69 on Coroico-La Paz road, 16 13'33"S, $67^{\circ} 49^{\prime} 20^{\prime \prime} \mathrm{W}, 2700 \mathrm{~m}$, Yungas secondary cloud forest, corticolous, 23 May 2011, $A F 21483$ \& OP (LPB); near Nogalani, on Coroico-La Paz road, $16^{\circ} 12^{\prime} 57^{\prime \prime} \mathrm{S}$, $67^{\circ} 49^{\prime} 15^{\prime \prime} \mathrm{W}, 2168 \mathrm{~m}$, Yungas secondary cloud forest, corticolous, 23 May 2011, AF $21392 \&$ \&P (LPB). 


\section{Graphis duplicata Ach.}

Fifth record of the species in Bolivia (Flakus et al. 2013; Kukwa et al. 2013).

Specimens examined. BOliVia. Dept. Tarija. Prov. Aniceto Arce, Filo de Sidras, $22^{\circ} 14^{\prime} 50^{\prime \prime} \mathrm{S}$, $64^{\circ} 33^{\prime} 28^{\prime \prime} \mathrm{W}, 1064 \mathrm{~m}$, Tucumano-Boliviano submontane forest, corticolous, 22 Nov. 2010, AF 18602.2 (LPB).

\section{${ }^{*}$ Graphis globosa (Fée) Spreng.}

Previously known from Brazil and from the type collection with no locality given (Lücking et al. 2009).

Specimens examined. BOliVia. Dept. Tarija. Prov. Aniceto Arce, Filo de Sidras, $22^{\circ} 14^{\prime} 50^{\prime \prime} \mathrm{S}, 64^{\circ} 33^{\prime} 28^{\prime \prime} \mathrm{W}$, $1064 \mathrm{~m}$, Tucumano-Boliviano submontane forest, corticolous, 22 Nov. 2010, AF 18614 (KRAM, LPB, UGDA).

${ }^{*}$ Graphis haleana R. C. Harris

Previously recorded in Brazil and the USA (Florida) (Lücking et al. 2009).

Specimens examined. BOliVia. Dept. Santa Cruz. Prov. José Miguel de Velasco, PN Noel Kempff Mercado, Savana de los Fierros near Florida, $14^{\circ} 36^{\prime} 11^{\prime \prime}$, $61^{\circ} 52^{\prime} 24^{\prime \prime} \mathrm{W}, 205 \mathrm{~m}$, savannah, corticolous, $15 \mathrm{Dec}$. 2009, AF 15628 \& PR (KRAM, LPB).

\section{Graphis illinata Eschw.}

In Bolivia reported previously from three localities (Flakus \& Wilk 2006; Schiefelbein et al. 2014).

Specimens examined. BOliVia. Dept. La Paz. Prov. Franz Tamayo, APOLOBAMBA, near Paujeyuyo, $15^{\circ} 02^{\prime} 46^{\prime \prime} \mathrm{S}, 68^{\circ} 27^{\prime} 46^{\prime \prime} \mathrm{W}, 960 \mathrm{~m}$, Sub-Andean Amazon forest close to plantation, corticolous, 12 Nov. 2014, MK 14515 (LPB, UGDA).

\section{Graphis librata Knight}

Second locality of the species in Bolivia (Schiefelbein et al. 2014).

Specimens examined. BOliVia. Dept. Santa Cruz. Prov. Cordillera, KAA-IYA, near Peto Blanco, park guard's station, $18^{\circ} 51^{\prime} 36^{\prime \prime} \mathrm{S}, 60^{\circ} 18^{\prime} 00^{\prime \prime} \mathrm{W}, 290 \mathrm{~m}$, transition Chiquitano-Chaqueño forest, corticolous, 5 Dec. 2011, $A F 23700$ (KRAM, LPB).

\section{Graphis lineola Ach.}

Second Bolivian record of the species (Kukwa et al. 2013).

Specimens examined. BOliVia. Dept. Santa Cruz. Prov. Cordillera, between Tucavaca and Roboré, $18^{\circ} 36^{\prime} 11^{\prime \prime} \mathrm{S}, 59^{\circ} 53^{\prime} 06^{\prime \prime} \mathrm{W}, 320 \mathrm{~m}$, transition ChiquitanoChaqueño forest, corticolous, 4 Dec. 2011, AF 23540.1 (KRAM, LPB).

\section{${ }^{*}$ Graphis marginata Raddi}

Pantropical species known in the Neotropics from Brazil and Costa Rica (Lücking et al. 2008, 2009).

Specimen examined. BOlivia. Dept. La Paz. Prov. Nor Yungas, service station near Chuspipata, on Coroico-La Paz road, $16^{\circ} 17^{\prime} 09^{\prime \prime} \mathrm{S}, 67^{\circ} 51^{\prime} 00^{\prime \prime} \mathrm{W}, 3228 \mathrm{~m}$, Yungas cloud forest with Ericaceae, corticolous, 24 May 2011, AF $21652 \& O P$ (LPB).

Graphis mexicana (Hale) Kalb, Lücking \& Lumbsch

Second locality of the species in Bolivia (Kukwa et al. 2013).

Specimens examined. BOliVia. Dept. Tarija. Prov. Aniceto Arce, Filo de Sidras, $22^{\circ} 14^{\prime} 50^{\prime \prime} \mathrm{S}$, 643'ㅇ' $28^{\prime \prime} \mathrm{W}, 1064 \mathrm{~m}$, Tucumano-Boliviano submontane forest, muscicolous, 22 Nov. 2010, AF 18434.1, 18458,18471 (KRAM, LPB).

\section{Graphis parilis Kremp.}

In Bolivia reported previously from one locality (Kukwa et al. 2013).

Specimens examined. BOLIVIA. Dept. Tarija. Prov. Aniceto Arce, Filo de Sidras, $22^{\circ} 14^{\prime} 50^{\prime \prime} \mathrm{S}, 64^{\circ} 33^{\prime} 28^{\prime \prime} \mathrm{W}$, $1064 \mathrm{~m}$, Tucumano-Boliviano submontane forest, corticolous, 22 Nov. 2010, $A F$ 18567.1 (KRAM, LPB).

${ }^{*}$ Graphis pilarensis Cáceres \& Lücking

Previously known only from Brazil (Cáceres 2007).

Specimens examined. BOliVia. Dept. La Paz. Prov. Bautista Saavedra, $28 \mathrm{~km}$ from Charazani, on Charazani-Apolo road, $15^{\circ} 12^{\prime} 43^{\prime \prime} \mathrm{S}, 68^{\circ} 47^{\prime} 25^{\prime \prime} \mathrm{W}$, $1650 \mathrm{~m}$, Yungas montane forest, open area with boulders, corticolous, 18 May 2011, $A F 21063$ \& $O P$ (LPB). 


\section{Graphis puiggarii (Müll. Arg.) Lücking}

Fifth locality of the species in Bolivia (Schiefelbein et al. 2014).

Specimen examined. BOlivia. Dept. La Paz. Prov. Franz Tamayo, between Apolo and Mapiri, $14^{\circ} 41^{\prime} 50^{\prime \prime} \mathrm{S}, 68^{\circ} 25^{\prime} 07^{\prime \prime} \mathrm{W}, 1490 \mathrm{~m}$, savannah with scattered trees, 22 Nov. 2011, corticolous, AF 23007 (LPB).

\section{${ }^{*}$ Graphis striatula (Ach.) Spreng.}

Pantropical species reported in the Neotropics from Brazil, Colombia, Costa Rica, the Dominican Republic and Puerto Rico (Staiger 2002; Cáceres 2007; Lücking et al. 2008, 2009; Sipman et al. 2008).

Specimen examined. BOLIVIA. Dept. Tarija. Prov. Aniceto Arce, Filo de Sidras, $22^{\circ} 14^{\prime} 50^{\prime \prime} \mathrm{S}, 64^{\circ} 33^{\prime} 28^{\prime \prime} \mathrm{W}$, $1064 \mathrm{~m}$, Tucumano-Boliviano submontane forest, corticolous, 22 Nov. 2010, AF 18567.2 (LPB).

\section{Graphis tenella Ach.}

In Bolivia reported previously from one locality (Kukwa et al. 2013).

Specimens examined. BOLIVIA. Dept. CochaBAMBA. Prov. Carrasco, CARRASCO, near Sehuencas, $17^{\circ} 30^{\prime} 12^{\prime \prime} \mathrm{S}, 65^{\circ} 16^{\prime} 30^{\prime \prime} \mathrm{W}, 2220 \mathrm{~m}$, Yungas cloud forest, corticolous, 21 July 2008, $A F$ 10459, MK \& PR (KRAM, LPB).

\section{${ }^{* * *}$ Graphis tenoriensis Chaves \& Lücking}

Previously known only from Costa Rica (Lücking et al. 2008, 2009).

Specimens examined. BOliVia. Dept. Santa Cruz. Prov. Cordillera, KAA-IYA, near Peto Blanco, park guard's station, $18^{\circ} 56^{\prime} 26^{\prime \prime} \mathrm{S}, 60^{\circ} 22^{\prime} 39^{\prime \prime} \mathrm{W}, 340 \mathrm{~m}$, Chiquitano forest, corticolous, 5 Dec. 2011, AF 23626 (KRAM, LPB, UGDA).

\section{Graphis vestitoides (Fink) Staiger}

In Bolivia reported previously from one locality (Kukwa et al. 2013).

Specimens examined. BOliVia. Dept. Beni. Prov. Yacuma, E. B. BENI, near la Estación Biológica, $14^{\circ} 51^{\prime} 07^{\prime \prime} \mathrm{S}, 66^{\circ} 20^{\prime} 23^{\prime \prime} \mathrm{W}, 175 \mathrm{~m}$, island of lowland Amazon forest among savannah, corticolous, 6 Nov. 2010, AF 17900 \& F. Saavedra (LPB).

\section{${ }^{*}$ Hemithecium oryzaeforme (Fée) Staiger}

Previously known from Ecuador and from the type specimen with no locality given (Staiger 2002; Nöske et al. 2007).

Specimens examined. BOLIVIA. Dept. Santa Cruz. Prov. Cordillera, KAA-IYA, near Peto Blanco, park guard's station, $18^{\circ} 51^{\prime} 36^{\prime \prime} \mathrm{S}, 60^{\circ} 18^{\prime} 00^{\prime \prime} \mathrm{W}, 290 \mathrm{~m}$, transition Chiquitano-Chaqueño forest, corticolous, 5 Dec. 2011, AF 23699 (KRAM, LPB).

Heterodermia comosa (Eschw.) Follman \& Rédon

In Bolivia known previously from only a few recent records (Flakus et al. 2012a, 2014 and literature cited therein). New for Muñecas Province and Área Natural de Manejo Integrado Nacional Apolobamba.

Specimens examined. BOliVia. Dept. La Paz. Prov. Muñecas, APOLOBAMBA, above Camata, close to Charazani-Paujeyuyo road, $15^{\circ} 14^{\prime} 18^{\prime \prime} \mathrm{S}, 68^{\circ} 45^{\prime} 09^{\prime \prime} \mathrm{W}$, $1800 \mathrm{~m}$, open area with shrubs, on shrub, 13 Nov. 2014, MK 14603 (LPB, UGDA).

\section{Heterodermia leucomela subsp. boryi (Fée)}

Swinscow \& Krog

In Bolivia known from several records (Feuerer \& Sipman 2005; Flakus et al. 2012a, 2014). New for Bautista Saavedra Province and Área Natural de Manejo Integrado Nacional Apolobamba.

Specimen examined. BOliVia. Dept. La Paz. Prov. Bautista Saavedra, APOLOBAMBA, near La Curva, close to Charazani, $15^{\circ} 06^{\prime} 33^{\prime \prime} \mathrm{S}, 69^{\circ} 01^{\prime} 51^{\prime \prime} \mathrm{W}$, 3634 m, open area with shrubs, on Polylepis sp., 14 Nov. 2014, MK 14654 (LPB).

\section{Heterodermia parva Moberg}

Second locality of the species in Bolivia (Flakus et al. 2012a).

Specimens examined. BOliVia. Dept. CochaBAmba. Prov. Tiraque, CARRASCO, close to Antenas Sillar, $17^{\circ} 12^{\prime} 55^{\prime \prime} \mathrm{S}, 65^{\circ} 42^{\prime} 35^{\prime \prime} \mathrm{W}, 3750 \mathrm{~m}$, open area with shrubs, corticolous, 30 Nov. 2014, $A F 26014$ (KRAM, LPB, herb. Flakus).

\section{Heterodermia squamulosa Degel.}

In Bolivia only recently reported from six localities (Flakus et al. 2013). New for Muñecas 
Province and Área Natural de Manejo Integrado Nacional Apolobamba.

Specimens examined. BOliVia. Dept. La Paz. Prov. Muñecas, APOLOBAMBA, above Camata, close to Charazani-Paujeyuyo road, $15^{\circ} 14^{\prime} 18^{\prime \prime} \mathrm{S}, 68^{\circ} 45^{\prime} 09^{\prime \prime} \mathrm{W}$, $1800 \mathrm{~m}$, open area with shrubs, on rock, 13 Nov. 2014, MK 14614 (LPB).

Hypogymnia subphysodes (Kremp.) Filson

In Bolivia reported previously from three localities (Flakus et al. 2011, 2012a).

Specimens eXamined. BOLIVIA. Dept. CochaBAmba. Prov. Carrasco, CARRASCO, La Cumbre, El Camino de las Nubes, $17^{\circ} 17^{\prime} 46^{\prime \prime} \mathrm{S}, 65^{\circ} 43^{\prime} 56^{\prime \prime} \mathrm{W}, 4100 \mathrm{~m}$, Páramo Yungueño, on rock, 19 Aug. 2012, MK 11781 (LPB); DePt. La PAz. Prov. Bautista Saavedra, APOLOBAMBA, la Cumbre close to Charazani-Pelechuco road, $14^{\circ} 48^{\prime} 09^{\prime \prime} \mathrm{S}, 69^{\circ} 10^{\prime} 51^{\prime \prime} \mathrm{W}, 4850 \mathrm{~m}$, open high Andean vegetation, saxicolous, 15 Nov. 2014, $A F 25008$ (LPB).

Hypotrachyna columbiensis (Zahlbr.) Divakar, Crespo, Sipman, Elix \& Lumbsch

Everniastrum columbiense (Zahlbr.) Sipman

Second Bolivian locality of the species (Flakus et al. 2011).

Specimens examined. BOlivia. Dept. La Paz. Prov. Larecaja, Jocollone, $15^{\circ} 37^{\prime} 35^{\prime \prime} \mathrm{S}, 68^{\circ} 41^{\prime} 21^{\prime \prime} \mathrm{W}$, $3545 \mathrm{~m}$, Páramo Yungueño vegetation, open built-up agricultural area, on soil, 14 May 2011, MK 8510 (LPB, UGDA).

\section{Hypotrachyna dentella (Hale \& Kurok.) Hale}

In Bolivia reported previously from two localities, both in Aniceto Arce Province (Dept. Tarija) (Flakus et al. 2012b; Divakar et al. 2013).

Specimens examined. BOliVia. Dept. Tarija. Prov. Burnet O'Connor, Lomas de la Soledad, road between Entre Ríos and Chiquiacá, $21^{\circ} 39^{\prime} 38^{\prime \prime} \mathrm{S}, 6^{\circ} 07^{\prime} 31^{\prime \prime} \mathrm{W}$, $1670 \mathrm{~m}$, Tucumano-Boliviano altimontano forest, 10 Aug. 2012, MK 11309 (LPB, UGDA).

Hypotrachyna nepalensis (Taylor) Divakar, Crespo, Sipman, Elix \& Lumbsch

Everniastrum nepalense (Taylor) Sipman

Second locality of the species in Bolivia (Flakus et al. 2011).
Specimens examined. BOliVia. Dept. La Paz. Prov. Nor Yungas, near Pongo, near Coroico-La Paz road, $16^{\circ} 19^{\prime} 28^{\prime \prime} \mathrm{S}, 67^{\circ} 57^{\prime} 21^{\prime \prime} \mathrm{W}, 3822 \mathrm{~m}$, Páramo Yungueño, on rock, 26 Nov. 2011, MK 10455 (LPB, UGDA).

\section{Hypotrachyna peruviana (Nyl.) Hale}

In Bolivia reported previously from four localities (Sipman et al. 2009; Flakus et al. 2011).

Specimens examined. BOliVia. Dept. CochaBAMBA. Prov. Carrasco, CARRASCO, near Sehuencas, $17^{\circ} 26^{\prime} 14^{\prime \prime} \mathrm{S}, 65^{\circ} 15^{\prime} 20^{\prime \prime} \mathrm{W}, 2150 \mathrm{~m}$, Yungas cloud forest, on bryophytes, 18 Aug. 2012, MK 11746 (LPB, UGDA).

\section{Hypotrachyna physcioides (Nyl.) Hale}

Reported several times from Bolivia (e.g., Feuerer et al. 1998; Sipman et al. 2009; Flakus et al. 2011; Divakar et al. 2013). Second record from Tarija department. New for Burnet O'Connor Province.

Specimens examined. BOliVia. Dept. Tarija. Prov. Burnet O'Connor, Lomas de la Soledad, road between Entre Ríos and Chiquiacá, $21^{\circ} 39^{\prime} 38^{\prime \prime} \mathrm{S}$, $64^{\circ} 07^{\prime} 31^{\prime \prime} \mathrm{W}, 1670 \mathrm{~m}$, Tucumano-Boliviano altimontano forest, on rock, 10 Aug. 2012, MK 11307 (LPB, UGDA).

\section{${ }^{*}$ Hypotrachyna protochlorina Sipman, Elix} \& T. H. Nash

Previously known only from Brazil (Sipman et al. 2009).

Specimens examined. BOliVia. Dept. Tarija. Prov. Burnet O'Connor, Lomas de la Soledad, road between Entre Ríos and Chiquiacá, $21^{\circ} 39^{\prime} 38^{\prime \prime} \mathrm{S}$, $64^{\circ} 07^{\prime} 31^{\prime \prime} \mathrm{W}, 1670 \mathrm{~m}$, Tucumano-Boliviano altimontano forest, on rock, 10 Aug. 2012, MK 11320 (LPB, UGDA).

\section{${ }^{*}$ Hypotrachyna sinuosella Elix, T. H. Nash}

\section{\& Sipman}

Previously reported from Colombia, Costa Rica and Venezuela (Sipman et al. 2009).

Specimens examined. BOliVia. Dept. La Paz. Prov. Bautista Saavedra, APOLOBAMBA, near La Curva, close to Charazani, $15^{\circ} 06^{\prime} 15^{\prime \prime} \mathrm{S}, 69^{\circ} 00^{\prime} 32^{\prime \prime} \mathrm{W}$, $3928 \mathrm{~m}$, open area with shrubs, on rock, 14 Nov. 2014, MK 14629 (LPB, UGDA). 


\section{*Hypotrachyna tariensis Elix}

Previously reported in the Neotropics from Colombia, Costa Rica, Ecuador and Peru, and elsewhere from Papua New Guinea (Sipman et al. 2009).

Specimens examined. BOliVia. Dept. La Paz. Prov. Bautista Saavedra, APOLOBAMBA, near la Curva, close to Charazani, $15^{\circ} 06^{\prime} 15^{\prime \prime} \mathrm{S}, 69^{\circ} 00^{\prime} 32^{\prime \prime} \mathrm{W}$, $3928 \mathrm{~m}$, open area with shrubs, on shrub, 14 Nov. 2014, MK 14630 (LPB, UGDA).

Hypotrachyna vexans (W. L. Culb. \& C. F. Culb.) Divakar, Crespo, Sipman, Elix \& Lumbsch

Everniastrum vexans (W. L. Culb. \& C. F. Culb.) Sipman

In Bolivia reported previously from numerous localities (Flakus et al. 2011, 2012a). New for Santa Cruz department.

Specimens examined. BOlivia. Dept. Santa Cruz. Prov. Florida, AMBORÓ, above La Yunga, senda Los Helechos, $18^{\circ} 03^{\prime} 30^{\prime \prime} \mathrm{S}, 6^{\circ} 54^{\prime} 36^{\prime \prime} \mathrm{W}, 2330 \mathrm{~m}$, Yungas cloud forest, on tree, 8 June 2011, MK 9836 (LPB, UGDA).

Lobariella pallida (Hook. f.) Moncada \& Lücking

In Bolivia reported previously from five localities (Kukwa et al. 2014). New for Muñecas Province and Área Natural de Manejo Integrado Nacional Apolobamba.

Specimens examined. BOliVia. Dept. La Paz. Prov. Muñecas, APOLOBAMBA, above Camata, close to Charazani-Paujeyuyo road, $15^{\circ} 14^{\prime} 35^{\prime \prime} \mathrm{S}, 68^{\circ} 45^{\prime} 09^{\prime \prime} \mathrm{W}$, 1900 m, open area with shrubs, on shrub, 13 Nov. 2014, MK 14580 (LPB, UGDA).

\section{Lobariella subcrenulata Moncada \& Lücking}

Second record of the species in Bolivia (Kukwa et al. 2014).

Specimens examined. BOlivia. Dept. La Paz. Prov. Muñecas, APOLOBAMBA, above Camata, close to Charazani-Paujeyuyo road, $15^{\circ} 14^{\prime} 18^{\prime \prime} \mathrm{S}, 68^{\circ} 45^{\prime} 09^{\prime \prime} \mathrm{W}$, 1800 , open area with shrubs, on shrub, 13 Nov. 2014, MK 14601 (LPB, UGDA).

Megalaria versicolor (Flot.) Fryday \& Lendemer

In Bolivia reported by Flakus (2008) and Flakus et al. (2012a, 2015). New for Carrasco Province.
Specimens examined. BOlivia. Dept. CochaBAmba. Prov. Carrasco, CARRASCO, near Río Lopez Mendoza, $17^{\circ} 30^{\prime} 25^{\prime \prime} \mathrm{S}, 65^{\circ} 16^{\prime} 51^{\prime \prime} \mathrm{W}, 2248 \mathrm{~m}$, lower montane Yungas cloud forest, corticolous, 27 Nov. 2014, AF 25639 (LPB); Sehuencas close to Monte Punku, 17 $30^{\prime} 19^{\prime \prime} \mathrm{S}, 65^{\circ} 16^{\prime} 40^{\prime \prime} \mathrm{W}, 222 \mathrm{~m}$, lower montane Yungas cloud forest, corticolous, 27 Nov. 2014, AF 25700 (KRAM, LPB).

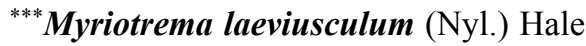

Previously reported only from Costa Rica, Cuba and the USA (Sipman et al. 2012; Esslinger 2015).

Specimens examined. BOlivia. Dept. Santa Cruz. Prov. Cordillera, on road from Tucavaca to Roboré, $18^{\circ} 37^{\prime} 56^{\prime \prime} \mathrm{S}, 59^{\circ} 36^{\prime} 50^{\prime \prime} \mathrm{W}, 220 \mathrm{~m}$, transition Chiquitano-Chaqueño forest, corticolous, 4 Dec. 2011, AF 23472 (KRAM, LPB).

\section{Nephroma helveticum Ach.}

In Bolivia known previously from only a few records (Nylander 1861; Kukwa et al. 2014). New for Tiraque Province and Parque Nacional Carrasco.

Specimen examined. BOliVia. Dept. CochaBAmba. Prov. Tiraque, CARRASCO, E of Ichuloma, old guard's camp, $17^{\circ} 18^{\prime} 23^{\prime \prime} \mathrm{S}, 65^{\circ} 45^{\prime} 60^{\prime \prime} \mathrm{W}, 3360 \mathrm{~m}$, open area with shrubs, corticolous, 1 Dec. 2014, AF 26028 (LPB).

\section{Parmelia warmingii Vain.}

A poorly known species, in Bolivia reported recently from two localities (Flakus et al. 2015).

Specimens examined. BOliVia. Dept. La Paz. Prov. Bautista Saavedra, APOLOBAMBA, near La Curva, close to Charazani, $15^{\circ} 06^{\prime} 15^{\prime \prime} \mathrm{S}, 69^{\circ} 00^{\prime} 32^{\prime \prime} \mathrm{W}$, $3928 \mathrm{~m}$, open area with shrubs, on rock, 14 Nov. 2014, MK 14617 (LPB, UGDA).

\section{${ }^{*}$ Parmeliella isidiopannosa P. M. Jørg.}

Previously known from Costa Rica, Ecuador and Venezuela (Jørgensen 2003; Jørgensen \& Arvidsson 2004).

Specimens examined. BOliVia. Dept. La Paz. Prov. Muñecas, APOLOBAMBA, above Camata, close to Charazani-Paujeyuyo road, $15^{\circ} 14^{\prime} 35^{\prime \prime} \mathrm{S}, 68^{\circ} 45^{\prime} 09^{\prime \prime} \mathrm{W}$, $1900 \mathrm{~m}$, open area with shrubs, on rock, 13 Nov. 2014, MK 14615 (LPB, UGDA). 


\section{Parmotrema aberrans (Vain.) Hale}

In Bolivia reported previously from three localities (Kukwa et al. 2012; Flakus et al. 2014).

Specimens examined. BOliVia. Dept. La Paz. Prov. Franz Tamayo, between Apolo and Mapiri, $14^{\circ} 41^{\prime} 50^{\prime \prime} \mathrm{S}, 68^{\circ} 25^{\prime} 07^{\prime \prime} \mathrm{W}, 1490 \mathrm{~m}$, savannah with scattered trees, corticolous, 22 Nov. 2011, MK 10129 (LPB, UGDA); between Apolo and Mapiri, $14^{\circ} 50^{\prime} 51^{\prime \prime} \mathrm{S}$, $68^{\circ} 21^{\prime} 38^{\prime \prime} \mathrm{W}, 1490 \mathrm{~m}$, savannah with scattered trees, shrubs, along streams, corticolous, 23 Nov. 2011, MK 10279, 10291a, 10295 (LPB, UGDA).

\section{Parmotrema argentinum (Kremp.) Hale}

In Bolivia known previously from three localities (Kukwa et al. 2012).

Specimens examined. BOliVia. Dept. La Paz. Prov. Franz Tamayo, between Apolo and Mapiri, $14^{\circ} 41^{\prime} 50^{\prime \prime} \mathrm{S}, 68^{\circ} 25^{\prime} 07^{\prime \prime} \mathrm{W}, 1490 \mathrm{~m}$, savannah with scattered trees, corticolous, 22 Nov. 2011, MK 10155, 10231 (LPB, UGDA); Prov. Nor Yungas, Coroico, near Sol y Luna hotel, $16^{\circ} 11^{\prime} 56^{\prime \prime} \mathrm{S}, 67^{\circ} 43^{\prime} 26^{\prime \prime} \mathrm{W}, 1870 \mathrm{~m}$, gardens with trees, on tree, 13 Dec. 2011, MK 10798 (LPB, UGDA); Dept. Tarija. Prov. Burnet O'Connor, TARIQUÍA, near Salinas, $21^{\circ} 49^{\prime} 15^{\prime \prime} \mathrm{S}, 64^{\circ} 12^{\prime} 44^{\prime \prime} \mathrm{W}$, $1400 \mathrm{~m}$, Tucumano-Boliviano montano forest, corticolous, 10 Aug. 2012, MK 11199 (LPB, UGDA).

\section{Parmotrema cetratum (Ach.) Hale}

In Bolivia reported several times previously (Herzog 1922; Feuerer et al. 1998; Flakus et al. 2011, 2012a, 2015). New for Área Natural de Manejo Integrado Nacional Apolobamba.

Specimens examined. BOLIVIA. Dept. La Paz. Prov. Bautista Saavedra, APOLOBAMBA, near La Curva, close to Charazani, $15^{\circ} 06^{\prime} 33^{\prime \prime} \mathrm{S}, 69^{\circ} 01^{\prime} 51^{\prime \prime} \mathrm{W}$, 3634 m, open area with shrubs, on Polylepis sp., 14 Nov. 2014, MK 14644 (LPB, UGDA).

\section{Parmotrema commensuratum (Hale) Hale}

Second record of the species in Bolivia (Flakus et al. 2014).

The specimen contained atranorin, norlobaridone, loxodin, salazinic and consalazinic acids. The last two substances were not reported by Hale (1977) and Flakus et al. (2014).

Specimens examined. BOliVia. Dept. La Paz. Prov. Nor Yungas, Coroico, near Sol y Luna hotel, $16^{\circ} 11^{\prime} 56^{\prime \prime} \mathrm{S}, 67^{\circ} 43^{\prime} 26^{\prime \prime} \mathrm{W}, 1870 \mathrm{~m}$, gardens with trees, corticolous, 13 Dec. 2011, MK 10780 (LPB, UGDA).

\section{*Parmotrema conferendum Hale}

Previously reported from Argentina, Colombia, the USA and Venezuela (Hale 1977; Sipman et al. 2008).

Specimens examined. BOlivia. Dept. La Paz. Prov. Nor Yungas, above Coroico, alt. 1600 m, on Juniperus sp., 30 Sept. 1997, S. Stab LB182a (B); Coroico, near Sol y Luna hotel, $16^{\circ} 11^{\prime} 56^{\prime \prime} \mathrm{S}, 67^{\circ} 43^{\prime} 26^{\prime \prime} \mathrm{W}, 1870 \mathrm{~m}$, gardens with trees, on rock, 13 Dec. 2011, MK 10809 (LPB, UGDA).

\section{Parmotrema conformatum (Vain.) Hale}

Reported previously from Bolivia only once (Flakus et al. 2014).

Specimens examined. BOliVia. Dept. La Paz. Prov. Franz Tamayo, between Apolo and Mapiri, $14^{\circ} 41^{\prime} 50^{\prime \prime} \mathrm{S}, 68^{\circ} 25^{\prime} 07^{\prime \prime} \mathrm{W}, 1490 \mathrm{~m}$, savannah with scattered trees, corticolous, 22 Nov. 2011, MK 10119, 10122 (LPB, UGDA); Prov. Larecaja, between Tipuani and Guanay, $15^{\circ} 31^{\prime} 24^{\prime \prime} \mathrm{S}, 67^{\circ} 58^{\prime} 06^{\prime \prime} \mathrm{W}, 1200 \mathrm{~m}$, Preandean Amazon forest, corticolous, 24 Nov. 2011, MK 10437 (LPB, UGDA); Prov. Nor Yungas, Coroico, near Sol y Luna hotel, $16^{\circ} 11^{\prime} 56^{\prime \prime} \mathrm{S}, 67^{\circ} 43^{\prime} 26^{\prime \prime} \mathrm{W}, 1870 \mathrm{~m}$, garden, corticolous, 13 Dec. 2011, MK 10775 (LPB, UGDA).

\section{Parmotrema cristiferum (Taylor) Hale}

Third record of the species in Bolivia (Kukwa et al. 2012).

Specimens examined. BOLIVIA. Dept. Santa Cruz. Prov. Cordillera, KAA-IYA, near Peto Blanco (park guard's station), 18 56'26"S, 60²2'39"W, $340 \mathrm{~m}$, Chiquitano forest, corticolous, 5 Dec. 2011, MK 10645 (LPB, UGDA).

\section{Parmotrema dilatatum (Vain.) Hale}

In Bolivia reported previously from five localities (Hale 1965; Kukwa et al. 2012; Flakus et al. 2014). New for Bautista Saavedra Province, and the first contemporary record from Franz Tamayo Province.

Specimens examined. BOliVia. Dept. La Paz. Prov. Bautista Saavedra, $28 \mathrm{~km}$ from Charazani town, on road between Charazani and Apolo, $15^{\circ} 12^{\prime} 43^{\prime \prime} \mathrm{S}$, $68^{\circ} 47^{\prime} 25^{\prime \prime} \mathrm{W}, 1650 \mathrm{~m}$, Yungas montane forest, open area with boulders, on rock, 18 May 2011, MK 8883 
(LPB, UGDA); Prov. Franz Tamayo, between Apolo and Mapiri, $15^{\circ} 01^{\prime} 50^{\prime \prime} \mathrm{S}, 68^{\circ} 18^{\prime} 33^{\prime \prime} \mathrm{W}, 1120 \mathrm{~m}$, Preandean Amazon forest, corticolous, 23 Nov. 2011, MK 10307 (LPB, UGDA).

\section{Parmotrema disparile (Nyl.) Hale}

In Bolivia known previously from only one record (Flakus et al. 2014).

Specimens examined. BOliVia. Dept. Tarija. Prov. Burnet O'Connor, TARIQUÍA, near Salinas, $21^{\circ} 49^{\prime} 15^{\prime \prime} \mathrm{S}, 64^{\circ} 12^{\prime} 44^{\prime \prime} \mathrm{W}, 1400 \mathrm{~m}$, Tucumano-Boliviano montano forest, corticolous, 10 Aug. 2012, MK $11240 a$ (LPB, UGDA); Prov. Burnet O'Connor, $26 \mathrm{~km}$ from Entre Ríos, near Soledad, $21^{\circ} 39^{\prime} 52^{\prime \prime} \mathrm{S}, 64^{\circ} 07^{\prime} 22^{\prime \prime} \mathrm{W}$, $1700 \mathrm{~m}$, Tucumano-Boliviano montano forest, corticolous, 11 Aug. 2012, MK 11341 (LPB, UGDA).

\section{Parmotrema eciliatum (Nyl.) Hale}

Fifth locality of the species in Bolivia (Kukwa et al. 2012).

Specimens examined. BOliVia. Dept. TariJa. Prov. Burnet O'Connor, Lomas de la Soledad, road between Entre Ríos and Chiquiacá, $21^{\circ} 39^{\prime} 38^{\prime \prime} \mathrm{S}, 64^{\circ} 07^{\prime} 31^{\prime \prime} \mathrm{W}$, $1670 \mathrm{~m}$, Tucumano-Boliviano altimontano forest, corticolous, 10 Aug. 2012, MK 11258 (LPB, UGDA).

\section{*Parmotrema enteroxanthum Hale}

Previously known from Colombia and Venezuela (Hale 1977; Sipman et al. 2008).

Specimens examined. BOLIVIA. Dept. Santa Cruz. Prov. Cordillera, KAA-IYA, near Peto Blanco, $18^{\circ} 51^{\prime} 36^{\prime \prime} \mathrm{S}, 60^{\circ} 18^{\prime} 00^{\prime \prime} \mathrm{W}, 310 \mathrm{~m}$, transition ChiquitanoChaqueño forest, corticolous, 5 Dec. 2011, MK 10669 (LPB, UGDA).

\section{Parmotrema flavescens (Kremp.) Hale}

Second locality of the species in Bolivia (Kukwa et al. 2012).

Specimens examined. BOliVia. Dept. La Paz. Prov. Muñecas, APOLOBAMBA, above Camata, close to Charazani-Paujeyuyo road, $15^{\circ} 14^{\prime} 18^{\prime \prime} \mathrm{S}, 68^{\circ} 45^{\prime} 09^{\prime \prime} \mathrm{W}$, 1800 m, open area with shrubs, on rock, 13 Nov. 2014, MK 14609 (LPB, UGDA).

\section{Parmotrema flavomedullosum Hale}

In Bolivia reported previously from two localities (Kukwa et al. 2012).
Specimens examined. BOliVia. Dept. La Paz. Prov. Franz Tamayo, between Apolo and Mapiri, $14^{\circ} 41^{\prime} 50^{\prime \prime} \mathrm{S}, 68^{\circ} 25^{\prime} 07^{\prime \prime} \mathrm{W}, 1490 \mathrm{~m}$, savannah with scattered trees, corticolous, 22 Nov. 2011, MK 10213 (LPB, UGDA); Prov. Nor Yungas, Coroico, near Sol y Luna hotel, $1870 \mathrm{~m}, 16^{\circ} 11^{\prime} 56^{\prime \prime} \mathrm{S}, 67^{\circ} 43^{\prime} 26^{\prime \prime} \mathrm{W}$, garden, on tree, 13 Dec. 2011, MK 10786 (LPB, UGDA); DePt. TARIJA. Prov. Burnet O'Connor, TARIQUÍA, near Salinas, $21^{\circ} 49^{\prime} 15^{\prime \prime} \mathrm{S}, 64^{\circ} 12^{\prime} 44^{\prime \prime} \mathrm{W}, 1400 \mathrm{~m}$, Tucumano-Boliviano montano forest, corticolous, 10 Aug. 2012, MK 11211 (LPB, UGDA).

Parmotrema gardneri (C. W. Dodge) Sérus.

In Bolivia reported previously by Kukwa et al. (2012) and Flakus et al. (2014). New for Franz Tamayo and Cordillera provinces.

Specimens examined. BOliVia. Dept. CochaBAmba. Prov. Carrasco, CARRASCO, Koricaza, $17^{\circ} 33^{\prime} 21^{\prime \prime} \mathrm{S}, 65^{\circ} 16^{\prime} 29^{\prime \prime} \mathrm{W}, 2950 \mathrm{~m}$, Páramo Yungueño, corticolous, 18 Aug. 2012, MK 11700 (LPB, UGDA); Dept. La Paz. Prov. Franz Tamayo, between Apolo and Mapiri, $14^{\circ} 41^{\prime} 50^{\prime \prime} \mathrm{S}, 68^{\circ} 25^{\prime} 07^{\prime \prime} \mathrm{W}, 1490 \mathrm{~m}$, savannah with scattered trees, corticolous, 22 Nov. 2011, MK 10117 (LPB, UGDA); Dept. Santa Cruz. Prov. Cordillera, SSW of Roboré, $18^{\circ} 36^{\prime} 11^{\prime \prime} \mathrm{S}, 59^{\circ} 53^{\prime} 06^{\prime \prime} \mathrm{W}$, $320 \mathrm{~m}$, transition Chiquitano-Chaqueño forest, corticolous, 4 Dec. 2011, MK 10580 (LPB, UGDA).

*Parmotrema laciniellum (Ferraro \& Elix) Blanco, Crespo, Divakar, Elix \& Lumbsch

Previously known from Argentina (Ferraro \& Elix 2000).

Specimens examined. BOlivia. Dept. Tarija. Prov. Burnet O'Connor, $90 \mathrm{~km}$ from Tarija on the way to Entre Ríos, $21^{\circ} 25^{\prime} 32^{\prime \prime} \mathrm{S}, 64^{\circ} 19^{\prime} 05^{\prime \prime} \mathrm{W}, 2250 \mathrm{~m}$, Tucumano-Boliviano altimontano forest, with Alnus acuminata and Podocarpus sp., on Podocarpus sp., 9 Aug. 2012, MK 11161 (LPB, UGDA).

\section{Parmotrema mellissii (Dodge) Hale}

In Bolivia reported previously by Kukwa et al. (2012) and Flakus et al. (2014). New for Franz Tamayo and Burnet O'Connor provinces.

Specimens examined. BOliVia. Dept. La Paz. Prov. Franz Tamayo, between Apolo and Mapiri, $14^{\circ} 41^{\prime} 50^{\prime \prime} \mathrm{S}, 68^{\circ} 25^{\prime} 07^{\prime \prime} \mathrm{W}, 1490 \mathrm{~m}$, savannah with scattered trees, corticolous, 22 Nov. 2011, MK 10236, 10215 (LPB, UGDA); Prov. Nor Yungas, Coroico, near Sol y Luna hotel, $1870 \mathrm{~m}, 16^{\circ} 11^{\prime} 56^{\prime \prime} \mathrm{S}, 67^{\circ} 43^{\prime} 26^{\prime \prime} \mathrm{W}$, garden, 
corticolous, 13 Dec. 2011, MK 10744, 10818 (LPB, UGDA); Dept. Tarija. Prov. Burnet O'Connor, Lomas de la Soledad, road between Entre Ríos and Chiquiacá, $21^{\circ} 39^{\prime} 38^{\prime \prime} \mathrm{S}, 64^{\circ} 07^{\prime} 31^{\prime \prime} \mathrm{W}, 1670 \mathrm{~m}$, Tucumano-Boliviano altimontano forest, corticolous, 10 Aug. 2012, MK 11317 (LPB, UGDA).

\section{Parmotrema mesotropum (Müll. Arg.) Hale}

In Bolivia reported previously by Hale (1965) and Flakus et al. (2012a, 2014). New for Parque Nacional y Área Natural de Manejo Integrado KAA-IYA del Gran Chaco.

In the studied material some specimens morphologically similar to $P$. mesotropum but containing caperatic acid instead of constipatic acid complex were also found. Another such sample was reported previously (as $P$. cf. mesotropum) by Flakus et al. (2012a). This material may represent an undescribed species but the lack of molecular data (specimens too old for standard DNA analysis) prevents establishment of its phylogenetic position at present.

Specimens examined. BOLIVIA. Dept. Santa Cruz. Prov. Cordillera, KAA-IYA, near Peto Blanco (park guard's station), $18^{\circ} 56^{\prime} 26^{\prime \prime} \mathrm{S}, 60^{\circ} 22^{\prime} 39^{\prime \prime} \mathrm{W}, 340 \mathrm{~m}$, Chiquitano forest, corticolous, 5 Dec. 2011, MK 10642 (LPB, UGDA).

SPECIMENS CONTAINING CAPERATIC ACID EXAMINED. BOLIVIA. DePt. La Paz. Prov. Abel Iturralde, Tumupasa, $14^{\circ} 03^{\prime} \mathrm{S}, 67^{\circ} 49^{\prime} \mathrm{W}, 400 \mathrm{~m}$, on wood, 3 March 1987 , S. Stab LB26 (B); $46 \mathrm{~km}$ por camino de Ixiamas hacia Alto Madidi, $13^{\circ} 38^{\prime} \mathrm{S}, 68^{\circ} 26^{\prime} \mathrm{W}, 300 \mathrm{~m}$, bosque siempreverde, epiphytic, 13 Aug. 1997, K. Bach 392, 393 et al. (B); Prov. Sud Yungas, above Yanacachi, $3200 \mathrm{~m}$, on stone, 3 March 1987, S. Stab LB62 (B).

\section{Parmotrema mirandum (Hale) Hale}

In Bolivia reported previously from a single locality (Kukwa et al. 2012).

Specimens examined. BOliVia. Dept. La Paz. Prov. Franz Tamayo, between Apolo and Mapiri, $14^{\circ} 41^{\prime} 50^{\prime \prime} \mathrm{S}, 68^{\circ} 25^{\prime} 07^{\prime \prime} \mathrm{W}, 1490 \mathrm{~m}$, savannah with scattered trees, corticolous, 22 Nov. 2011, MK 10114 (LPB, UGDA); Prov. Franz Tamayo, between Apolo and Mapiri, $13 \mathrm{~km}$ from Apolo, $14^{\circ} 50^{\prime} 51^{\prime \prime} \mathrm{S}, 68^{\circ} 21^{\prime} 38^{\prime \prime} \mathrm{W}$, $1490 \mathrm{~m}$, savannah with scattered trees, shrubs, along streams, corticolous, 23 Nov. 2011, MK 10292 (LPB, UGDA).

\section{Parmotrema mordenii (Hale) Hale}

Second locality of the species in Bolivia (Flakus et al. 2014).

Specimens examined. BOliVia. Dept. Tarija. Prov. Burnet O'Connor, TARIQUÍA, near Salinas, $21^{\circ} 49^{\prime} 15^{\prime \prime} \mathrm{S}, 64^{\circ} 12^{\prime} 44^{\prime \prime} \mathrm{W}, 1400 \mathrm{~m}$, Tucumano-Boliviano montano forest, saxicolous, 10 Aug. 2012, MK 11242 (LPB, UGDA).

\section{Parmotrema neotropicum Kurok.}

In Bolivia reported previously from a few localities (Kukwa et al. 2012; Flakus et al. 2013). Second locality in Tamayo Province.

Specimens examined. BOliVia. Dept. La Paz. Prov. Franz Tamayo, between Apolo and Mapiri, $14^{\circ} 41^{\prime} 50^{\prime \prime} \mathrm{S}, 68^{\circ} 25^{\prime} 07^{\prime \prime} \mathrm{W}, 1490 \mathrm{~m}$, savannah with scattered trees, corticolous, 22 Nov. 2011, MK 10179 (LPB, UGDA).

* Parmotrema pilosum (Stizenb.) Krog \& Swinscow

Previously reported in South America from Argentina, Chile, Ecuador and Uruguay, and elsewhere from Africa (Hale 1976c; Krog \& Swinscow 1981; Calvelo \& Liberatore 2002).

Specimens examined. BOliVia. Dept. La Paz. Prov. Franz Tamayo, between Apolo and Mapiri, $14^{\circ} 41^{\prime} 50^{\prime \prime} \mathrm{S}, 68^{\circ} 25^{\prime} 07^{\prime \prime} \mathrm{W}, 1490 \mathrm{~m}$, savannah with scattered trees, corticolous, 22 Nov. 2011, MK 10172, 10230 (LPB, UGDA).

\section{Parmotrema rampoddense (Nyl.) Hale}

Rarely reported in Bolivia (Hale 1965; Flakus et al. 2011, 2012a).

Specimens examined. BOliVia. Dept. La Paz. Prov. Nor Yungas, Coroico, near Hotel Sol y Luna, $1870 \mathrm{~m}, 16^{\circ} 11^{\prime} 56^{\prime \prime} \mathrm{S}, 67^{\circ} 43^{\prime} 26^{\prime \prime} \mathrm{W}$, garden, corticolous, 13 Dec. 2011, MK 10814 (LPB, UGDA); DePt. SANTA Cruz. Prov. Cordillera, KAA-IYA, $259 \mathrm{~km}$ on road from Tucavaca to Roboré, $18^{\circ} 32^{\prime} 37^{\prime \prime} \mathrm{S}, 60^{\circ} 30^{\prime} 03^{\prime \prime} \mathrm{W}, 370 \mathrm{~m}$, transition Chiquitano-Chaqueño forest, corticolous, 3 Dec. 2011, MK 10500a (LPB, UGDA).

\section{${ }^{*}$ Parmotrema recipiendum (Nyl.) Hale}

Previously reported from Argentina, Brazil, Colombia and Paraguay (Hale 1965; Calvelo \& Liberatore 2002; Sipman et al. 2008).

Specimens examined. BOLIVIA. Dept. Cocha- 
Bamba. Prov. Ayopaya, $20 \mathrm{~km}$ de Cocapata hacia Cotacajes, $16^{\circ} 46^{\prime} \mathrm{S}, 66^{\circ} 44^{\prime} \mathrm{W}, 2000 \mathrm{~m}$, bosque semideciduo disturbado, corticolous, 15 May 1997, K. Bach 84 et al. (B).

Parmotrema reticulatum (Taylor) M. Choisy s.l.

In Bolivia reported previously several times (Nylander 1859; Herzog 1922; Feuerer et al. 1998; Flakus et al. 2011, 2012b, 2014). New for Franz Tamayo and Burnet O'Connor provinces.

Specimens examined. BOLIVIA. Dept. La Paz. Prov. Franz Tamayo, between Apolo and Mapiri, $14^{\circ} 41^{\prime} 50^{\prime \prime} \mathrm{S}, 68^{\circ} 25^{\prime} 07^{\prime \prime} \mathrm{W}, 1490 \mathrm{~m}$, savannah with scattered trees, corticolous, 22 Nov. 2011, MK 10234, 10131 (LPB, UGDA); ibid., $14^{\circ} 50^{\prime} 51^{\prime \prime} \mathrm{S}, 68^{\circ} 21^{\prime} 38^{\prime \prime} \mathrm{W}, 1490 \mathrm{~m}$, 23 Nov. 2011, $M K 10274$ (LPB, UGDA); DePt. TARIJA. Prov. Burnet O'Connor, near San Diego, $21^{\circ} 26^{\prime} 28^{\prime \prime} \mathrm{S}$, $64^{\circ} 14^{\prime} 37^{\prime \prime} \mathrm{W}, 1620 \mathrm{~m}$, Tucumano-Boliviano altimontano forest, on rock, 9 Aug. 2012, $M K 11190$ (LPB, UGDA); TARIQUÍA, near Salinas, $21^{\circ} 49^{\prime} 15^{\prime \prime} \mathrm{S}, 64^{\circ} 12^{\prime} 44^{\prime \prime} \mathrm{W}$, $1400 \mathrm{~m}$, Tucumano-Boliviano montano forest, on rock, 10 Aug. 2012, MK 11235 (LPB).

\section{Parmotrema robustum (Degel.) Hale}

In Bolivia reported previously by Kukwa et al. (2012) and Flakus et al. (2014). New for Tarija department.

Specimens examined. BOliVia. Dept. Tarija. Prov. Burnet O'Connor, Lomas de la Soledad, road between Entre Ríos and Chiquiacá, $21^{\circ} 39^{\prime} 38^{\prime \prime} \mathrm{S}, 64^{\circ} 07^{\prime} 31^{\prime \prime} \mathrm{W}$, $1670 \mathrm{~m}$, Tucumano-Boliviano altimontano forest, corticolous, 10 Aug. 2012, MK 11283 (LPB, UGDA).

\section{${ }^{*}$ Parmotrema ruptum (Lynge) Hale ex DePriest} \& Hale

Previously known from Brazil, Paraguay and Uruguay (Spielmann 2009 and literature cited therein).

Specimens examined. BOLIVIA. Dept. La Paz. Prov. Muñecas, APOLOBAMBA, above Camata, close to Charazani-Paujeyuyo road, $15^{\circ} 14^{\prime} 18^{\prime \prime} \mathrm{S}, 68^{\circ} 45^{\prime} 09^{\prime \prime} \mathrm{W}$, $1800 \mathrm{~m}$, open area with shrubs, on rock, 13 Nov. 2014, MK 14602, 14611 (LPB, UGDA).

\section{Parmotrema sancti-angelii (Lynge) Hale}

In Bolivia reported previously from a single locality only (Kukwa et al. 2012).

Specimens examined. BOliVia. Dept. La Paz.
Prov. Nor Yungas, Coroico, Hotel Sol y Luna, $16^{\circ} 11^{\prime} 56^{\prime \prime} \mathrm{S}, 67^{\circ} 43^{\prime} 26^{\prime \prime} \mathrm{W}, 1870 \mathrm{~m}$, garden, on rock, 13 Dec. 2011, $M K$ 10825, 10828 (LPB, UGDA).

\section{Parmotrema subarnoldii (Abbayes) Hale}

Second locality in Bolivia (Kukwa et al. 2012).

Specimens examined. BOliVia. Dept. La Paz. Prov. Nor Yungas, near Nogalani, on Coroico-La Paz road, $16^{\circ} 12^{\prime} 57^{\prime \prime} \mathrm{S}, 67^{\circ} 49^{\prime} 15^{\prime \prime} \mathrm{W}, 2168 \mathrm{~m}$, Yungas secondary cloud forest, corticolous, 23 May 2011, MK 9141 (LPB, UGDA).

\section{Parmotrema subisidiosum (Müll. Arg.) Hale}

In Bolivia reported previously from two localities (Kukwa et al. 2012).

Specimens examined. BOliVia. Dept. La Paz. Prov. Franz Tamayo, between Apolo and Mapiri, $14^{\circ} 41^{\prime} 50^{\prime \prime} \mathrm{S}, 68^{\circ} 25^{\prime} 07^{\prime \prime} \mathrm{W}, 1490 \mathrm{~m}$, savannah with scattered trees, corticolous, 22 Nov. 2011, MK 10163, 10165 (LPB, UGDA).

Parmotrema tinctorum (Despr. ex Nyl.) Hale

Reported from Bolivia by Kukwa et al. (2012) and Flakus et al. (2014). New for Reserva Nacional de Flora y Fauna Tariquía.

Specimens examined. BOliVia. Dept. Tarija. Prov. Burnet O'Connor, TARIQUÍA, near Salinas, $21^{\circ} 49^{\prime} 15^{\prime \prime} \mathrm{S}, 64^{\circ} 12^{\prime} 44^{\prime \prime} \mathrm{W}, 1400 \mathrm{~m}$, Tucumano-Boliviano montano forest, on rock, 10 Aug. 2012, MK 11195 , 11214, 11234 (LPB, UGDA).

Peltigera dolichorrhiza (Nyl.) Nyl.

Reported from Bolivia by Herzog (1922) and Kukwa et al. (2014). New for Carrasco Province.

Specimens examined. BOLIVIA. Dept. Cochabamba. Prov. Carrasco, CARRASCO, near Phaqcha, $17^{\circ} 27^{\prime} 13^{\prime \prime}$ S, $65^{\circ} 16^{\prime} 44^{\prime \prime} \mathrm{W}, 2850 \mathrm{~m}$, montane cloud forest, close to river, on soil, 20 July 2008, MK 6181 (LPB, UGDA).

Peltigera fibrilloides (Gyeln.) Vitik.

Feuerer and Sipman (2005) reported only one Bolivian locality of the species.

Specimens examined. BOlivia. Dept. CochaBAMBA. Prov. Carrasco, CARRASCO, near Sehuencas, $17^{\circ} 30^{\prime} 12^{\prime \prime} \mathrm{S}, 65^{\circ} 16^{\prime} 30^{\prime \prime} \mathrm{W}$, alt. $2220 \mathrm{~m}$, montane cloud forest, along road and close to settlement, on soil, 21 July 2008, MK 6379 (LPB, UGDA). 
Peltigera laciniata (G. Merr.) Gyeln.

In Bolivia known previously from several localities (Gyelnik 1930; Vitikainen 1994; Feuerer et al. 1998; Kukwa et al. 2014). New for Larecaja Province.

Specimens examined. BOliVia. Dept. La Paz. Prov. Larecaja, Titilaya, $15^{\circ} 39^{\prime} 55^{\prime \prime} \mathrm{S}, 68^{\circ} 42^{\prime} 51^{\prime \prime} \mathrm{W}, 2773$ $\mathrm{m}$, transition Youngas forest-Valles Secos vegetation, on soil, 14 May 2011, MK 8444 (LPB, UGDA).

Peltigera rufescens (Weiss) Humb.

In Bolivia reported previously by Nylander (1859), Rusby (1896), Feuerer et al. (1998) and Kukwa et al. (2014). New for Área Natural de Manejo Integrado Nacional Apolobamba.

Specimens examined. BOliVia. Dept. La Paz. Prov. Franz Tamayo, APOLOBAMBA, near Puyo Puyo, $14^{\circ} 56^{\prime} 55^{\prime \prime} \mathrm{S}, 6^{\circ} 07^{\prime} 58^{\prime \prime} \mathrm{W}, 4795 \mathrm{~m}$, high Andean open vegetation, on soil, 5 July 2010, $A F 17682 \& P R$ (LPB, KRAM).

\section{Peltigera ulcerata Müll. Arg.}

In Bolivia reported previously by Feuerer et al. (1998) and Kukwa et al. (2014). New for Oruro and Tarija departments, Franz Tamayo Province and Área Natural de Manejo Integrado Nacional Apolobamba.

Specimens examined. BOliVia. Dept. La Paz, Prov. Franz Tamayo, APOLOBAMBA, near Puyo Puyo, $14^{\circ} 56^{\prime} 55^{\prime \prime} \mathrm{S}, 6^{\circ} 07^{\prime} 58^{\prime \prime} \mathrm{W}, 4795 \mathrm{~m}$, high Andean open vegetation, on ground, 5 July 2010, AF 17693 \& PR (LPB); DePt. Oruro, Prov. Sajama, SAJAMA, Jecha K'ala, $18^{\circ} 09^{\prime} 52^{\prime \prime}$ S, 68 $49^{\circ} 08^{\prime \prime} \mathrm{W}, 4184$ m, Puna Sureña, pajonales vegetation, on soil, 20 June 2010, AF 16692 \& $P R$ (LPB, KRAM); Dept. TariJa. Prov. Aniceto

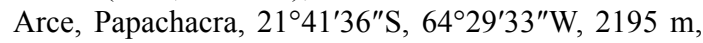
Tucumano-Boliviano montane forest, on soil, 26 Nov. 2010, AF 19800 \& $J Q$ (LPB).

\section{Phaeophyscia hispidula (Ach.) Essl.}

Fourth locality in Bolivia (Flakus et al. 2013).

Specimens examined. BOliVia. Dept. La Paz. Prov. Muñecas, APOLOBAMBA, above Camata, close to Charazani-Paujeyuyo road, $15^{\circ} 14^{\prime} 18^{\prime \prime} \mathrm{S}, 68^{\circ} 45^{\prime} 09^{\prime \prime} \mathrm{W}$, $1800 \mathrm{~m}$, open area with shrubs, on rock, 13 Nov. 2014, MK 14608, 14612 (LPB, UGDA).

\section{Phyllobaeis imbricata (Hook.) Kalb \& Gierl}

In Bolivia found previously at several localities (Flakus et al. 2012a and literature cited therein). New for Franz Tamayo Province, and the first modern record for Larecaja Province.

Specimens examined. BOLIVIA. Dept. La Paz. Prov. Franz Tamayo, near Correo, $14^{\circ} 59^{\prime} 06^{\prime \prime} \mathrm{S}, 68^{\circ} 26^{\prime} 09^{\prime \prime} \mathrm{W}$, $1630 \mathrm{~m}$, Yungas montane forest, open area near road, on rock, 17 May 2011, MK 8786 (LPB, UGDA); Prov. Larecaja, Jocollone, $15^{\circ} 37^{\prime} 35^{\prime \prime} \mathrm{S}, 68^{\circ} 41^{\prime} 21^{\prime \prime} \mathrm{W}, 3545 \mathrm{~m}$, Páramo Yungueño vegetation, open agricultural area, on soil, 14 May 2011, MK 8538 (LPB, UGDA).

\section{Platygramme caesiopruinosa (Fée) Fée}

In Bolivia reported previously from nine localities (Flakus et al. 2013; Kukwa et al. 2013; Schiefelbein et al. 2014). New for Chapare Province.

Specimens examined. BOliViA. Dept. CochaBAmBA. Prov. Chapare, near Incachaca, $17^{\circ} 12^{\prime} 54^{\prime \prime} \mathrm{S}$, $65^{\circ} 49^{\prime} 30^{\prime \prime} \mathrm{W}, 2028 \mathrm{~m}$, Yungas cloud forest near road, 7 July 2009, AF 13023 (LPB).

\section{Pleopsidium chlorophanum (Wahlenb.) Zopf}

In Bolivia reported previously only by $\mathrm{Ny}$ lander (1861).

Specimens examined. BOliVia. Dept. La Paz. Prov. Larecaja, la Cumbre Ancoruma, close to SorataMapiri road, $14^{\circ} 43^{\prime} 09^{\prime \prime} \mathrm{S}, 68^{\circ} 35^{\prime} 55^{\prime \prime} \mathrm{W}, 4650 \mathrm{~m}$, open high Andean vegetation, saxicolous, 21 Nov. 2014, AF 25489 (KRAM, LPB).

\section{Pseudephebe pubescens (L.) M. Choisy}

Fifth record of the species in Bolivia (Feuerer \& Sipman 2005; Flakus \& Wilk 2006; Flakus et al. 2011).

Specimen examined. BOlivia. Dept. CochaBAMBA. Prov. Tiraque, CARRASCO, Diente del Diablo, close to Antenas Sillar-Cotany Alto road, $17^{\circ} 16^{\prime} 55^{\prime \prime} \mathrm{S}$, $65^{\circ} 44^{\prime} 14^{\prime \prime} \mathrm{W}, 4335 \mathrm{~m}$, open high Andean vegetation, saxicolous, 2 Dec. 2014, $A F 26053$ (LPB).

Pseudocyphellaria crocata (L.) Vain.

Fourth locality of the species in Bolivia (Feuerer et al. 1998; Kukwa et al. 2014).

Specimens examined. BOLIVIA. Dept. CochaBAmba. Prov. Carrasco, CARRASCO, Sehuencas, 
$2220 \mathrm{~m}, 17^{\circ} 30^{\prime} 12^{\prime \prime} \mathrm{S}, 65^{\circ} 16^{\prime} 30^{\prime \prime} \mathrm{W}$, corticolous, 21 July 2008, $P R$ 758, $A F \& M K$ (LPB).

\section{Psiloparmelia distincta (Nyl.) Hale}

Numerous Bolivian records of the species (Flakus et al. 2015 and literature cited therein). New for Carrasco Province and Parque Nacional Carrasco.

Specimens examined. BOLIVIA. Dept. CochaBAMBA. Prov. Carrasco, CARRASCO, La Cumbre, El Camino de las Nubes, $17^{\circ} 17^{\prime} 46^{\prime \prime} \mathrm{S}, 65^{\circ} 43^{\prime} 56^{\prime \prime} \mathrm{W}, 4100 \mathrm{~m}$, Páramo Yungueño, on rock, 19 Aug. 2012, MK 11780 , 11797 (LPB, UGDA).

\section{Psiloparmelia flavobrunnea (Müll.Arg.) Elix} \& Nash

In Bolivia reported previously by Elix and Nash (1992) and Flakus et al. (2013). Second record for Bautista Saavedra Province.

Specimens examined. BOliVia. Dept. La Paz. Prov. Bautista Saavedra, APOLOBAMBA, near La Curva, close to Charazani, $15^{\circ} 06^{\prime} 15^{\prime \prime} \mathrm{S}, 6^{\circ} 00^{\prime} 32^{\prime \prime} \mathrm{W}, 3928 \mathrm{~m}$, open area with shrubs, on rock, 14 Nov. 2014, MK 14626 (LPB, as host in specimen of Lichenostigma sp.).

\section{*Pyxine pungens Zahlbr.}

Previously reported from Australia, Indonesia, Central and South America (Brazil, Costa Rica, Guyana, Paraguay, Venezuela) (Jungbluth \& Marcelli 2011).

Specimen examined. BOlivia. Dept. La Paz. Prov. Nor Yungas, near Pongo, near Coroico-La Paz road, $16^{\circ} 19^{\prime} 28^{\prime \prime} \mathrm{S}, 67^{\circ} 57^{\prime} 21^{\prime \prime} \mathrm{W}, 3822 \mathrm{~m}$, Páramo Yungueño, on twig, 26 Nov. 2011, MK 10450c (LPB).

Ramboldia russula (Ach.) Kalb, Lumbsch \& Elix

In Bolivia reported previously from a few localities; it is still under-collected (Nylander 1859; Kalb et al. 2008; Flakus et al. 2012a).

Specimens examined. BOliVia. Dept. La Paz. Prov. Franz Tamayo, between Apolo and Mapiri, $14^{\circ} 41^{\prime} 50^{\prime \prime} \mathrm{S}, 68^{\circ} 25^{\prime} 07^{\prime \prime} \mathrm{W}, 1490 \mathrm{~m}$, savannah with scattered trees, corticolous, 22 Nov. 2011, MK 10235 (LPB, UGDA); Dept. Santa Cruz. Prov. Cordillera, SSW of Roboré, $18^{\circ} 36^{\prime} 11^{\prime \prime} \mathrm{S}, 59^{\circ} 53^{\prime} 06^{\prime \prime} \mathrm{W}, 320 \mathrm{~m}$, transition Chiquitano-Chaqueño forest, corticolous, 4 Dec. 2011, MK $10563 b$ (LPB, UGDA).

\section{Reimnitzia santensis (Tuck.) Kalb}

Second Bolivian record of the species (Schiefelbein et al. 2014).

Specimens examined. BOlivia. Dept. Santa Cruz. Prov. Cordillera, near Peto Blanco, park guard's station, $18^{\circ} 48^{\prime} 25^{\prime \prime} \mathrm{S}, 60^{\circ} 14^{\prime} 54^{\prime \prime} \mathrm{W}, 330 \mathrm{~m}$, transition Chiquitano-Chaqueño forest, corticolous, 6 Dec. 2011, AF 23777 (KRAM, LPB).

\section{Siphula pteruloides Nyl.}

In Bolivia reported previously from four localities (Feuerer et al. 1998; Feuerer \& Sipman 2005; Flakus et al. 2014).

Specimen examined. BOlivia. Dept. CochaBAmba. Prov. Tiraque, CARRASCO, close to Antenas Sillar-Cotany Alto road, $17^{\circ} 14^{\prime} 22^{\prime \prime} \mathrm{S}, 65^{\circ} 43^{\prime} 07^{\prime \prime} \mathrm{W}$, $3870 \mathrm{~m}$, open area with shrubs, terricolous, 30 Nov. 2014, $A F 26023$ (LPB).

\section{Stereocaulon ramulosum (Sw.) Räusch}

The commonest species of the genus in Bolivia (e.g., Feuerer et al. 1998; Flakus et al. 2011, 2012a, 2015). New for Muñecas Province and Área Natural de Manejo Integrado Nacional Apolobamba.

Specimens examined. BOlivia. Dept. La Paz. Prov. Muñecas, APOLOBAMBA, above Camata, close to Charazani-Paujeyuyo road, $15^{\circ} 14^{\prime} 35^{\prime \prime} \mathrm{S}, 68^{\circ} 45^{\prime} 09^{\prime \prime} \mathrm{W}$, 1900 m, open area with shrubs, on rock, 13 Nov. 2014, $M K$ 14577, 14594 (LPB, UGDA).

\section{Stereocaulon tomentosum Fr.}

Fifth Bolivian record of the species (Feuerer et al. 1998; Flakus et al. 2011).

Specimens examined. BOliVia. Dept. La Paz. Prov. Bautista Saavedra, APOLOBAMBA, near La Curva, close to Charazani, $15^{\circ} 06^{\prime} 15^{\prime \prime} \mathrm{S}, 69^{\circ} 00^{\prime} 32^{\prime \prime} \mathrm{W}$, $3928 \mathrm{~m}$, open area with shrubs, on rock, 14 Nov. 2014, MK 14631 (LPB, UGDA).

\section{Stereocaulon vesuvianum Pers.}

In Bolivia recorded six times previously (Feuerer \& Sipman 2005; Flakus et al. 2011, 2012a, 2015). New for Área Natural de Manejo Integrado Nacional Apolobamba.

Specimens examined. BOlivia. Dept. La Paz. Prov. Bautista Saavedra, APOLOBAMBA, near La 
Curva, close to Charazani, $15^{\circ} 06^{\prime} 15^{\prime \prime} \mathrm{S}, 69^{\circ} 00^{\prime} 32^{\prime \prime} \mathrm{W}$, $3928 \mathrm{~m}$, open area with shrubs, on soil, 14 Nov. 2014, MK 14622, 14636 (LPB, UGDA).

\section{Teloschistes flavicans (Sw.) Norman}

In Bolivia reported previously from numerous localities (e.g., Feuerer et al. 1998; Søchting \& Fröden 2002; Flakus et al. 2012a, 2015). New for Muñecas Province and Área Natural de Manejo Integrado Nacional Apolobamba.

Specimens examined. BOlivia. Dept. La Paz. Prov. Bautista Saavedra, APOLOBAMBA, near la Curva, $15^{\circ} 06^{\prime} 33^{\prime \prime} \mathrm{S}, 6^{\circ} 01^{\prime} 51^{\prime \prime} \mathrm{W}, 3634 \mathrm{~m}$, open area with shrubs, on Polylepis sp., 14 Nov. 2014, MK 14652 (LPB, UGDA); Prov. Muñecas, APOLOBAMBA, above Camata, $15^{\circ} 14^{\prime} 35^{\prime \prime} \mathrm{S}, 68^{\circ} 45^{\prime} 09^{\prime \prime} \mathrm{W}, 1900 \mathrm{~m}$, open area with shrubs, on soil, 13 Nov. 2014, MK 14578 (LPB, UGDA).

Thamnolia papelillo R. Sant.

In Bolivia reported previously by Santesson (2004), Flakus (2008) and Flakus et al. (2012a, 2015). New for Tiraque Province and Área Natural de Manejo Integrado Nacional Apolobamba.

Specimens examined. BOliVia. Dept. CochaBAmba. Prov. Tiraque, CARRASCO, Cerro Juno, near Antenas, $17^{\circ} 19^{\prime} 27^{\prime \prime} \mathrm{S}, 65^{\circ} 41^{\prime} 39^{\prime \prime} \mathrm{W}, 4649 \mathrm{~m}$, open high Andean vegetation, terricolous, 29 Nov. 2014,

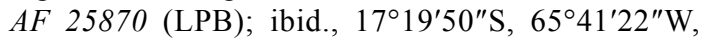
4662 m, $A F 25904$ (KRAM, LPB); DePt. La Paz. Prov. Bautista Saavedra, APOLOBAMBA, near La Curva, $15^{\circ} 06^{\prime} 33^{\prime \prime} \mathrm{S}, 69^{\circ} 01^{\prime} 51^{\prime \prime} \mathrm{W}, 3634 \mathrm{~m}$, open area with shrubs, terricolous, 14 Nov. 2014, $A F 24968$ (KRAM, LPB); la Cumbre, $14^{\circ} 48^{\prime} 09^{\prime \prime} \mathrm{S}, 69^{\circ} 10^{\prime} 51^{\prime \prime} \mathrm{W}, 4850 \mathrm{~m}$, open high Andean vegetation, terricolous, 15 Nov. 2014, $A F 25007$ (KRAM, LPB).

\section{Thamnolia vermicularis (Sw.) Schaer.}

A very common species in Bolivia at higher altitudes of the Andes (Nylander 1861; Menhofer 1992; Feuerer et al. 1998; Flakus \& Wilk 2006; Flakus 2008; Flakus et al. 2012a). New for Tiraque Province and Parque Nacional Carrasco.

Specimens examined. BOliVia. Dept. CochaBAmba. Prov. Tiraque, CARRASCO, Cerro Juno, $17^{\circ} 19^{\prime} 27^{\prime \prime} \mathrm{S}, 65^{\circ} 41^{\prime} 39^{\prime \prime} \mathrm{W}, 4649 \mathrm{~m}$, open high Andean vegetation, terricolous, 29 Nov. 2014, $A F 25874$

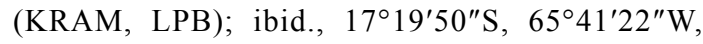

$4662 \mathrm{~m}, A F 25905$ (LPB); ibid., 17019'34"S, $65^{\circ} 42^{\prime} 13^{\prime \prime} \mathrm{W}, 4389 \mathrm{~m}, A F 25921$ (KRAM, LPB); Diente del Diablo, $17^{\circ} 16^{\prime} 55^{\prime \prime} \mathrm{S}, 65^{\circ} 44^{\prime} 14^{\prime \prime} \mathrm{W}, 4335 \mathrm{~m}$, open high Andean vegetation, terricolous, 2 Dec. 2014, $A F$ 26051 (LPB).

\section{Thelocarpon andicola Flakus \& Kukwa}

Second locality of this recently described species (Flakus \& Kukwa 2014).

Specimens examined. BOliVia. Dept. La Paz. Prov. Bautista Saavedra, APOLOBAMBA, near la Curva, close to Charazani, $15^{\circ} 06^{\prime} 15^{\prime \prime} \mathrm{S}, 69^{\circ} 00^{\prime} 32^{\prime \prime} \mathrm{W}$, $3928 \mathrm{~m}$, open area with shrubs, saxicolous, 14 Nov. 2014, AF 24955 (KRAM, LPB).

\section{${ }^{* *}$ Thelocarpon laureri (Flot.) Nyl.}

Previously known only from Africa (Canary Islands), Australia, Europe and North America (Salisbury 1966; Orange et al. 2009; McCarthy 2015).

Specimens examined. BOliVia. Dept. La Paz. Prov. Bautista Saavedra, APOLOBAMBA, near La Curva, close to Charazani, $15^{\circ} 06^{\prime} 15^{\prime \prime} \mathrm{S}, 69^{\circ} 00^{\prime} 32^{\prime \prime} \mathrm{W}$, $3928 \mathrm{~m}$, open area with shrubs, saxicolous, 14 Nov. 2014, AF 24954 (KRAM, LPB).

\section{* Xanthoparmelia substenophylloides Hale}

Previously reported in South America from Argentina, Brazil, Colombia, Peru, Uruguay and Venezuela, and elsewhere from South Africa (Hale 1989; Nash et al. 1995; Sipman et al. 2008).

Specimens examined. BOlivia. Dept. La Paz. Prov. Muñecas, APOLOBAMBA, above Camata, close to Charazani-Paujeyuyo road, $15^{\circ} 14^{\prime} 18^{\prime \prime} \mathrm{S}, 68^{\circ} 45^{\prime} 09^{\prime \prime} \mathrm{W}$, $1800 \mathrm{~m}$, open area with shrubs, on rock, 13 Nov. 2014, MK 14606 (LPB, UGDA).

\section{Yoshimuriella peltigera auct.}

In Bolivia reported previously from three localities (Kukwa et al. 2014).

Specimens examined. BOliviA. Dept. Beni. Prov. Ballivian, Puento Rio Quiquibey, $4 \mathrm{~km}$ hacia San Borja, $700 \mathrm{~m}$, bosque natural, sobre corteza, 14 July 1979, S. G. Beck 1736 (LPB); DePt. LA PAZ. Prov. Nor Yungas, COTAPATA, Chairo, $16^{\circ} 13^{\prime} 19.2^{\prime \prime} \mathrm{S}, 67^{\circ} 52^{\prime} 34^{\prime \prime} \mathrm{W}$, 1370 m, corticolous, 11 May 2006, PR 70, J. Beltrán, J. Torrez (LPB). 
Acknowledgements. Rosa I. Meneses Q. and the Herbario Nacional de Bolivia (Universidad Mayor de San Andrés and Museo Nacional de Historia Natural La Paz) are gratefully acknowledged for their generous cooperation, and the curators of the herbaria for the loan of specimens. Javier Quisbert, Oscar Plata, Pamela Rodriguez Flakus and all the staff of protected areas are thanked for their kind help during the field work. We thank the two anonymous reviewers for helpful remarks and suggestions on the manuscript. This research received support from the SYNTHESYS Project (http://www.synthesys.info/) which is financed by the European Community Research Infrastructure Action under the FP7 "Capacities" Programme (GB-TAF-4226), the National Centre for Research and Development (NCBiR) of Poland under the LIDER Programme for 2010-2013 (No. 92/L-1/09), and the W. Szafer Institute of Botany (Polish Academy of Sciences) and the University of Gdańsk through their statutory funds.

\section{REFERENCES}

Aнті T. 2000. Cladoniaceae. Fl. Neotrop. Monogr. 78: 1-362.

Benatti M. N. 2014. A review of the genus Bulbothrix Hale: the isidiate, lacinulate, sorediate and pustulate species with medullary gyrophoric, lecanoric and lobaric acids, together with a world key for the genus. Opuscula Philolichenum 13: $122-154$.

Benatti M. N. \& Elix J. A. 2012. The true identity of Bulbothrix goebelii (Zenker) Hale and the re-establishment of some of its synonyms as accepted species. Lichenologist 44(6): 813-826.

Bjerke J. W. \& Elvebakk A. 2004. Distribution of the lichen genus Flavocetraria (Parmeliaceae, Ascomycota) in the Southern Hemisphere. New Zealand J. Bot. 42: 647-656.

Bungartz F., Ziemmeck F., Yánez Ayabaca A., Nugra F. \& Apтroot A. 2013. CDF Checklist of Galapagos Lichenized Fungi. FCD Lista de especies de Hongos liquenizados Galápagos. [3 Dec. 2013]. http://checklists.datazone. darwinfoundation.org/true-fungi/lichens.

CÁCERES M. E. S. 2007. Corticolous crustose and microfoliose lichens of northeastern Brazil. IHW-Verlag und Verlagsbuchhandlung, München.

Calvelo S. \& Liberatore S. 2002. Catálogo de los líquenes de la Argentina. Kurtziana 29(2): 7-170.

Culberson C. F. \& Kristinsson H. 1970. A standardized method for the identification of lichen products. J. Chromatogr. 46: 85-93.

Divakar P. K., Crespo A., Núñez-Zapata J., Flakus A., Sipman H. J. M., Elix J. A. \& Lumbsch H. T. 2013. A molecular perspective on generic concepts in the $H y$ - potrachyna clade (Parmeliaceae, Ascomycota). Phytotaxa 132(1): 21-38.

Elix J. A. \& Nash T. H. 1992. A synopsis of the lichen genus Psiloparmelia (Ascomycotina, Parmeliaceae). Bryologist 95(4): 377-391.

Ertz D., Flakus A., Oset M., Sipman H. J. M. \& Kukwa M. 2015. A first assessment of lichenized Arthoniales in Bolivia with descriptions of two new species. Phytotaxa 217(1): 1-25.

ESSLINGER T. L. 2015. A cumulative checklist for the lichenforming, lichenicolous and allied fungi of the continental United States and Canada. North Dakota State University. [Most recent version (\#20) 19 April 2015]. http://www. ndsu.edu/pubweb/ esslinge/chcklst/chcklst7.htm

Fernández-Mendoza F. \& Printzen C. 2013. Pleistocene expansion of the bipolar lichen Cetraria aculeata into the Southern Hemisphere. Molec. Ecol. 22: 1961-1983.

Ferraro L. I. 2002. Contribution to the knowledge of foliicolous lichens of Bolivia. Mitt. Bot. Mus. Hamburg 30-32: 47-48.

Ferraro L. I. \& Elix J. A. 2000. A new species of Canomaculina (lichenized Ascomycotina, Parmeliaceae) from Argentina. Mycotaxon 74(2): 391-394.

Feuerer T. \& Sipman H. J. M. 2005. Additions to the lichenized and lichenicolous fungi of Bolivia. Herzogia 18: 139-144.

Feuerer T., Ahti T. \& Vitikainen O. 1998. Lichenological investigations in Bolivia. In: M. P. MARCELLI \& M. R. D. SEAWARD (eds), Lichenology in Latin America: history, current knowledge and applications, pp. 71-86. CETESB, Săo Paulo.

FlaKus A. 2008. Contributions to the knowledge of the lichen biota of Bolivia. 2. Polish Bot. J. 53(2): 145-153.

Flakus A. \& KuKwa M. 2014. The first squamulose Thelocarpon species (Thelocarpaceae, Ascomycota) discovered in the biological soil crusts in the Bolivian Andes. Phytotaxa 175(5): 281-286.

Flakus A. \& WiLK K. 2006. Contribution to the knowledge of the lichen biota of Bolivia. J. Hattori Bot. Lab. 99: 307-318.

Flakus A., Ahti T., Kukwa M. \& Wilk K. 2008. New and interesting records of Cladonia and their lichenicolous fungi from the Andean cloud forest in Bolivia. Ann. Bot. Fenn. 45: 448-454.

Flakus A., Oset M., Jablońska A., Rodriguez SaAVedra P. \& KuKwa M. 2011. Contribution to the knowledge of the lichen biota of Bolivia. 3. Polish Bot. J. 56(2): 159-183.

Flakus A., Etayo J., Schiefelbein U., Ahti T., Jablońska A., Oset M., Bach K., Rodriguez Flakus P. \& Kukwa M. 2012a. Contribution to the knowledge of the lichen biota of Bolivia. 4. Polish Bot. J. 53(2): 427-461.

Flakus A., Rodriguez SaAvedra P. \& KuKwa M. 2012 b. A new species and new combinations and records of 
Hypotrachyna and Remototrachyna from Bolivia. Mycotaxon 119: 157-166.

Flakus A., Sipman H. J. M., Bach K., Rodriguez Flakus P., Knudsen K., Аhti T., Schiefelbein U., Palice Z., Jablońska A., Oset M., Meneses Q. R. I. \& Kukwa M. 2013. Contribution to the knowledge of the lichen biota of Bolivia. 5. Polish Bot. J. 58(2): 697-733.

Flakus A., Sipman H. J. M., Rodriguez Flakus P., SchieFelbein U., JablońsKa A., Oset M. \& KuKWa M. 2014. Contribution to the knowledge of the lichen biota of Bolivia. 6. Polish Bot. J. 59(1): 63-83.

Flakus A., Sipman H. J. M., Rodriguez Flakus P., JablońsKa A., Oset M., Meneses Q. R. I. \& Kukwa M. 2015. Contribution to the knowledge of the lichen biota of Bolivia. 7. Polish Bot. J. 60(1): 81-98.

FrisCH A. 2006. The lichen family Thelotremataceae in Africa. A revision with special consideration of the taxa from Cameroon and Tanzania. Biblioth. Lichenol. 92: 3-370.

Gierl C. \& Kalb K. 1993. Die Flechtengattung Dibaeis. Eine Übersicht über die rosafrüchtigen Arten von Baeomyces sens. lat. nebst Anmerkungen zu Phyllobaeis gen. nov. Herzogia 9: 593-645.

Gilbert O. L. \& Hawksworth D. L. 2009. Catolechia Flot. (1850). In: C. W. Smith, A. Аptroot, B. J. Coppins, A. Fletcher, O. L. Gilbert, P. W. James \& P. A. WolseLEY (eds), The lichens of Great Britain and Ireland, p. 290. British Lichen Society, London.

Gyelnik V. 1930. Lichenes nonnulli novae criticique. Nyt Mag. Naturvidensk. 68: 269-270.

Hale M. E. 1965. A monograph of Parmelia subgenus Amphigymnia. Contr. U. S. Natl. Herb. 36(5): 193-358.

Hale M. E. 1976a. A monograph of the lichen genus Bulbothrix Hale (Parmeliaceae). Smithsonian Contr. Bot. 32: 1-29.

Hale M. E. 1976b. A monograph of the lichen genus Pseudoparmelia Lynge (Parmeliaceae). Smithsonian Contr. Bot. 31: 1-62.

Hale M. E. 1976c. A monograph of the lichen genus Parmelina Hale (Parmeliaceae). Smithsonian Contr. Bot. 33: 1-60.

Hale M. E. 1977. New species in the lichen genus Parmotrema Mass. Mycotaxon 5(2): 432-448.

Hale M. E. 1989. New species in he lichen genus Xanthoparmelia (Ascomycotina: Parmeliaceae). Mycotaxon 34(2): 541-564.

Hasselroth T. E. 1953. Nordliga lavar i syd-och Mellansverige. Acta Phytogeogr. Suec. 33: 1-200.

Herzog T. 1922. Beitrag zur Flechtenflora von Bolivia. Hedwigia 63: 263-268.

JørGENSEN P. M. 2003. Notes on some misunderstood, subtropical Parmeliella species. Bryologist 106(1): 121-129.

Jørgensen P. M. \& Arvidsson L. 2004. The lichen family Pannariaceae in Ecuador. Symb. Bot. Upsal. 34: 113-132.
Jungbluth P. \& Marcelli M. P. 2011. The Pyxine pungens complex in Săo Paulo State, Brazil. Bryologist 114(1): 166-177.

Kalb K., Staiger B., Elix J. A., Lange U. \& Lumbsch H. T. 2008. A new circumscription of the genus Ramboldia (Lecanoraceae, Ascomycota) based on morphological and molecular evidence. Nova Hedwigia 86(1-2): 23-42.

Krog H. \& Swinscow T. D. V. 1981. Parmelia subgenus Amphigymnia (lichens) in East Africa. Bull. Br. Mus. Nat. Hist., Bot. 9: 143-231.

Kukwa M., Bach K., Sipman H. J. M. \& Flakus A. 2012. Thirty-six species of the lichen genus Parmotrema (Lecanorales, Ascomycota) new to Bolivia. Polish Bot. J. 57(1): 243-257.

KuKwa M., Schiefelbein U. \& FlaKus A. 2013. A contribution to the lichen family Graphidaceae (Ostropales, Ascomycota) of Bolivia. Herzogia 26(2): 231-252.

Kukwa M., Sipman H. J. M., Etayo J., Bach K., GuzowKrzemińsKa B., JablońsKa A., OlszewsKa S., RodrIGuez Flakus P. \& Flakus A. 2014. The lichen order Peltigerales in Bolivia - the first assessment of the biodiversity. Herzogia 27(2): 321-345.

LÜCKING R. 2008. Foliicolous lichenized fungi. Fl. Neotrop. Monogr. 103: 1-867.

Lücking R., Aptroot A., Chaves J. L., Sipman H. J. M. \& UMAÑA L. 2007. A first assessment of the TICOLICHEN biodiversity inventory in Costa Rica: the genus Coccocarpia (Peltigerales: Coccocarpiaceae). Biblioth. Lichenol. 95: 429-457.

Lücking R., Chaves J. L., Sipman H. J. M., Umaña L. \& APTROOT A. 2008. A first assessment of the Ticolichen biodiversity inventory in Costa Rica: The genus Graphis, with notes on the genus Hemithecium (Ascomycota: Ostropales: Graphidaceae). Fieldiana, Bot. 46:1-131.

LückIng R., Archer A. W. \& Aptroot A. 2009. A world-wide key to the genus Graphis (Ostropales: Graphidaceae). $\mathrm{Li}$ chenologist 41(4): 363-452.

Lücking R., Dal-Forno M., Lawry J. D., Bungartz F., Holgado Rojas M. E., Hernández M. J. E., MarCelli M. P., Moncada B., Morales E. A., Nelsen M. P., Paz E., Salcedo L., Spielmann A. A., Wilk K., WillWolf S. \& YÁnez-Ayabaca A. 2013. Ten new species of lichenized Basidiomycota in the genera Dictyonema and Cora (Agaricales: Hygrophoraceae), with a key to all accepted genera and species in the Dictyonema clade. Phytotaxa 139(1): 1-38.

McCARthy P. M. 2015. Checklist of the lichens of Australia and its Island Territories.Australian Biological Resources Study, Canberra. [Version 1 July 2015]. http://www.anbg. gov.au/abrs/lichenlist/introduction.html.

Menhofer X. 1992. Die Vegetation des Wohngebietes der Kallawaya und des Hochlandes von Ulla Ulla in den bolivianischen Anden. Teil II. Phytocoenologia 20(3): 289-438. 
NASh III T. H., Gries C. \& Elix J. A. 1995. A revision of the lichen genus Xanthoparmelia in South America. Biblioth. Lichenol. 56: 1-158.

Nöske N. M., Mandl N. \& Sipman H. J. M. 2007. Lichens. Checklist Reserva Biológica San Francisco (Prov. Zamora-Chinchipe, S. Ecuador). Ecotropic. Monogr 4: 101-117.

NYLANDER W. 1859. Lichenes in regionibus exoticis quibusdam vigentes exponit synopticis enumerationibus. Ann. Sci. Nat. Bot. 11: 205-264.

NYLANDER W. 1861. Additamentum ad lichenographiam Andium Boliviensium. Ann. Sci. Nat. Bot. 15: 365-382.

Orange A., James P. W. \& White F. J. 2001. Microchemical methods for the identification of lichens. British Lichen Society, London.

Orange A., Watson M. F., James P. W. \& Moore D. M. 2009. Thelocarpon Nyl. (1853). In: C. W. Smith, A. Aptroot, B. J. Coppins, A. Fletcher, O. L. Gilbert, P. W. James \& P. A. WOLSELEY (eds), The lichens of Great Britain and Ireland, pp. 884-888. British Lichen Society, London.

Pérez Pérez R. E. \& Nash III T. H. 2010. The genus Canoparmelia in Mexico. Biblioth. Lichenol. 105: 225-238.

Rivas Plata E. \& LÜCKING R. 2013. High diversity of Graphidaceae (lichenized Ascomycota: Ostropales) in Amazonian Perú. Fung. Diversity 58(1): 13-32.

Rivas Plata E., Lücking R., Aptroot A., Sipman H. J. M., Chaves J. L., Umaña L. \& Lizano D. 2006. A first assessment of the Ticolichen biodiversity inventory in Costa Rica: the genus Coenogonium (Ostropales: Coenogoniaceae), with a world-wide key and checklist and a phenotype-based cladistic analysis. Fung. Diversity 23: 255-321.

Rivas Plata E., Lücking R., Sipman H. J. M., Mangold A., Kalb K. \& Lumbsch H. T. 2010. A world-wide key to the thelotremoid Graphidaceae, excluding the OcellulariaMyriotrema-Stegobolus clade. Lichenologist 42(2): 139-185.

RusBY H. H. 1896. An enumeration of the plant collected in Bolivia by Miguel Bang, with description of new genera and species. Part III. Mem. Torrey Bot. Club 6: 1-130.
SAlisbury G. 1966. A monograph of the lichen genus Thelocarpon Nyl. Lichenologist 3(2): 175-196.

SAntesson R. 2004. Two new species of Thamnolia. Symb. Bot. Upsal. 34(1): 393-397.

Schiefelbein U., Flakus A., Sipman H. J. M., Oset M. \& KuKwA M. 2014. A contribution to the lichen family Graphidaceae (Ostropales, Ascomycota) of Bolivia. 2. Polish Bot. J. 59(1): 85-96.

Sipman H. J. M., Hekking W. \& Aguirre-C. J. 2008. Checklist of Lichenized and Lichenicolous Fungi from Colombia. Biblioteca José Jerónimo Triana 20. Instituto de Ciencias Naturales, Facultad de Ciencias, Universidad Nacional de Colombia, Bogotá.

Sipman H. J. M., Elix J. A. \& Nash III T. H. 2009. Hypotrachyna (Parmeliaceae, lichenized Fungi). Fl. Neotrop. Monogr. 104: 1-173.

Sipman H. J. M., LÜcking R. Aptroot A. Chaves J. L., Kalb K. \& UMAÑA TENORIO L. 2012. A first assessment of the Ticolichen biodiversity inventory in Costa Rica and adjacent areas: the thelotremoid Graphidaceae (Ascomycota: Ostropales). Phytotaxa 55: 1-214.

Søchting U. \& FröDÉn P. 2002. Chemosyndromes in the lichen genus Teloschistes (Teloschistaceae, Lecanorales). Mycol. Progr. 1(3): 257-266.

Spielmann A. A. 2009. Estudos taxonômicos em Parmotrema s.1. (Parmeliaceae, Ascomycota liquenizados) com ácido salazínico. PhD Thesis, Instituto de Botânica, Săo Paulo.

Staiger B. 2002. Die Flechtenfamilie Graphidaceae. Studien in Richtung einer natürlicheren Gliederung. Biblioth. Lichenol. 85: 1-526.

Stenroos S., Pino-Bodas R., Weckman D. \& Ahti T. 2015. Phylogeny of Cladonia uncialis (Cladoniaceae, Lecanoromycetes) and its allies. Lichenologist 47(4): 215-231.

Vitikainen O. 1994. Notes on some Peltigera of the Neotropics. Acta Bot. Fenn. 150: 217-221. 